\title{
Antibiotics as a Feed Additive Promote Growth of Chickens by Modulating the Gut Microbiome
}

\author{
Xiangkai Li ( $\nabla$ xkli@lzu.edu.cn ) \\ Lanzhou University https://orcid.org/0000-0002-5704-9791

\section{Ying Wu} \\ Lanzhou University \\ Liang Peng \\ Lanzhou University \\ Rong Han \\ Lanzhou University \\ pengya Feng \\ Lanzhou University \\ Aman Khan \\ Lanzhou University \\ Pu Liu \\ Lanzhou University
}

\section{Research}

Keywords: Antibiotic, Gut microbiota, Metabonomic, Lipid and amid acid metabolism, Immune response

Posted Date: June 7th, 2021

DOl: https://doi.org/10.21203/rs.3.rs-571302/v1

License: (c) (i) This work is licensed under a Creative Commons Attribution 4.0 International License.

Read Full License 


\section{Abstract}

Background: Antibiotics widely used as growth promoters in the agricultural industry, but their mechanisms have not been fully explored. Antibiotics has a connection effect on the gut microbiome which plays a vital role in host metabolism and immune response. Here, we investigated the association of antibiotic and gut microbiome in broiler chicken.

Methods: Polymyxin (PMX) and amoxicillin (AMX) were selected as feed additives in broiler chicken, and gut bacterial composition assessed by quantitative real-time PCR and high-throughput sequencing of bacterial 16S rRNA. Metabolome and lipidomic of feces and serum samples were analyzed using liquid chromatograpy-tandem mass spectrometry (LC-MS). We further assessed changes in the microbiota and metabolism which underwent antibiotics treatment.

Results: The administration of antibiotic increasing, the average weight of chickens by up to $4.9 \%$ and altered number and structure of the intestinal microflora compared to the un-treated group. The bacterial component of gut microbiota in antibiotic groups was showed a lower prevalence of Firmicutes and Bacteroidetes phyla and higher prevalence of a high diversity (Proteobacteria). In metabolome of feces and serum, amino acid such as phenylalanine, valine, methionine and aspartic acid, and lipid metabolic (Lysolecithin, Oxalosuccinic acid and phosphatidylcholine) were all higher among obese chicks in antibiotics groups. Serum IgG (18.2\%-32.7\%) and intestinal secretory IgA (27.5\%-28.6\%) were decreased compared with control group, and immune associated metabolome in serum lipidomic were also fluctuated with the concentration of AMX and PMX.

Conclusions: The data suggested that the structure and quantity of intestinal flora has to affect amino acid and lipid metabolic homeostasis, and immune response, which can serve as a target in the prevention and treatment of metabolic disorders.

\section{Background:}

Antibiotic has been applied to animal husbandry which is rising globally at an unprecedented rate with the emergence and spread of antimicrobial resistance [1, 2]. The antibiotics used in livestock production were estimated to be 93,309 tons in 2017 and are expected to increase to 104,079 tons in 2030 globally, and about $73 \%$ of all antimicrobials used in animals were raised for food $[3,4]$. The initial purpose of antibiotic used in animal husbandry is for livestock diseases including gastrointestinal and respiratory tract infections with low cost and high cure efficiency $[5,6]$. However, low doses of antibiotics have been fed to large numbers of animals used for food production to increase weight gain [7]. For example, chlortetracycline can increase the growth rate of animal as much as 15\% [8]. Avoparcin, efrotomycin, lincomycin, penicillin $\mathrm{G}$ procaine, and virginiamycin have also improved the performance of animals in the farm, which contributed to large financial profits for producers and low cost for consumers $[9,10]$. Consequently, farmers prefer to use antibiotics, although many countries have banned the use of agricultural antibiotics [11]. The use of antibiotics is still in an upward trend in the future [12]. However, the mechanism that antibiotics can lead to obesity growth of animals is not very clear [13]. 
Gut microbiota has been recognized as an important factor in the maintenance of energy homeostasis and host immunity, contributing to the improved performance of the body [14]. Research showed that conventional mice had $40 \%$ more body fat than germ-free mice, regardless of food intake [15]. Meanwhile, colonization of germ-free mice with gut microbiota from conventional mice produced a significant increase in body weight and a $60 \%$ increase in body fat [16]. Besides, culture-based studies have indicated that intestinal bacteria tend to compete with the host for energy and amino acids [17]. As much as $6 \%$ of the net energy in pig diets can be lost due to bacterial utilization of glucose in the small intestine which reduces the energy available to the host epithelium [18]. Although the intestinal flora consumes part of the energy, it also synthesizes a variety of vitamins necessary for growth and development, such as vitamins, nicotinic acid and pantothenic acid $[19,20]$. Thus, a depleted microbiome could decrease the number of calories available for microbiome absorption and induce a hypoglycemic state, which maybe the potential role in energy storage [21]. For example, SCFA valerate, which is the main metabolic produce of microbiota, decreased 66-fold after antibiotic treatment [22, 23]. However, the basic questions relating to the effects of microbial production of intestine catabolites after quick antibiotics intervention remain mostly unanswered $[16,24]$.

Antibiotics are well known to result in the compositional and diversity changes of gut microbiota in animals [25]. The total bacterial community has induced a pronounced drop (-90\%) during antibiotic treatment per gram of intestinal content and has reached a minimum value after 11 days $[26,27]$. Evidence has also suggested that the antibiotic intervention flora in microbiota construction is inefficient. The prevalence of Bacteroidetes and Firmicutes were significantly reduced, while Proteobacteria was greatly increased in the guts of rats after antibiotic treatment, which is coincident with the development of obesity $[28,29,30]$. The major probiotic genera of gut microbiota is also changed, including Lactobacillus, Bifidobacterium and Helicobacter[31]. Besides, it is interesting to note that the growth-promoting effect of probiotics in livestock is less consistent than that observed with antibiotic supplementation, which may promote growth under the present of certain pathogens are presence [32]. For example, the Gram-positive facultative anaerobes, which are oxygen-tolerant and predominant in the small intestine, often contribute more than one toxic catabolite [33]. Hence, the depletion of gut microbiota rendered mice resistant to body weight loss, accompanied by the increase in fat mass [34]. So, whether antibiotics will reduce the energy consumption by changing intestinal flora is still unclear.

Based on abovementioned information, this study hypothesized that antibiotics can decrease the content and destroy the structure of intestinal flora to reduce energy consumption, making the host grow rapidly. But simultaneously, some beneficial factors also decrease, which are produced by gut microbiome, putting the host in an unhealthy growth state. Five broad-spectrum antibiotics, including Tylenol-tartrate, sulfadimidine, polymyxin, oxytetracycline and amoxicillin, were selected as feed additives in white feathered broilers as feed in normal-chow feed, which is widely used in animal husbandry [35, 36, 37]. In this model, the alteration of chicken metabolism has been demonstrated in different gut microbiota through short term antibiotic manipulation in high-dose doses. The results showed that the microbiome of chicken with antibiotic is depleted of all major bacterial phyla, as evidenced by decrease in cecum microbes. Antibiotic intervention has changed the vitamin digestion and absorption. In lipidomic, the 
insulin resistance, fat digestion and absorption, and cholesterol metabolism have been altered through the gut microbiota. Besides, some disease-related pathways are partly upregulated in the group with 800 $\mathrm{mg} / \mathrm{kg}$ of $A M X+P M X$. It reveals that antibiotic can change the deeply intertwined relationship among the gut microbiome through amino acid and fat metabolism and immune system, promoting energy accumulation.

\section{Results And Discussion}

\subsection{Antibiotics increased the growth of chicken}

The average daily gain (ADG = (the weight of day $42_{\text {nd }}$ the weight of day $\left.1_{\text {nd }}\right) / 41$ ) in AMX + PMX groups (up to $25.9 \%$ ) were highest among other antibiotic groups (14.3-23.4\%) within 42 days (Supplemental Figure $1 \mathrm{~A})$. For feed conversion ratio ( $\mathrm{FCR}=$ Live gain / feed consumption), the antibiotic groups especially $\mathrm{AMX}+\mathrm{PMX}_{1}$ group showed a marked increased (17.8-34.2\%) compared with the control group $(P<0.01)$ (Supplemental Figure 1C). No significant has been observed in food intake among all groups $(3.2 \%-21.1 \%)$ (Supplemental Figure 1B). Therefore, the combination of AMX + PMX was employed to further experiment (Figure 1A). The results showed that at $400 \mathrm{mg} / \mathrm{kg}$ of concentration, the growth of chickens reached to the maximum compared with that of the control group (4.9\%). However, after 800 $\mathrm{mg} / \mathrm{kg}$ of $\mathrm{AMX}+\mathrm{PMX}$ intervention, the weight was significantly decreased $2.5 \%$ than that in the control group (Figure 1B). It showed that the weight gain was increased with a certain dose of antibiotic intervention.

Antibiotics have been used as growth promoters at a subtherapeutic concentration in animals [38, 39]. Chlortetracycline and salinomycin (500 g/ton) produced a $9.8 \%$ higher ADG than the control [40]. Oxytetracycline $(100 \mathrm{mg} / \mathrm{kg})$ and florfenicol $(5 \mathrm{mg} / \mathrm{kg})$ also showed significant increase in the final body weight, which is consistent with obtained results [41]. However, the antibiotic group exhibit 10.6 higher ADG than control, low FCR than the control, which are unsimilar with early report [40]. These data indicated that AMX and PMX consider as a growth promoter for chicken broiler. The attribution of improvement of growth and feed efficiency as a result of dietary antibiotic supplementation is not clear, and needs further investigation [42].

\subsection{Health index, inflammation markers and oxidative stress indicators on chicken decreased with antibiotics}

The levels of AST, ALT, MDA, CAT and T-SOD were tested, which were similar with the control group $(\mathrm{P}<$ 0.01) (Figure 2A-E, Supplemental Figure 1). Histopathological analysis showed that a slight nuclear shrinkage in the liver of chicks were observed after being treated with $400 \mathrm{~g} / \mathrm{kg}$ of AMX + PMX (Figure $2 \mathrm{~F})$. Furthermore, under microscopical examination, hematoxylin-eosin staining of the adipose tissue sections in the antibiotic-treated chicken revealed that the distribution of mast cells was significantly more than that of the control group (Figure $2 \mathrm{G}$ ). The results showed that in the 35 days, both the 200 and 400 groups produced a 19.0 and $16.3 \%$ lower the ratio of liver/body weight than the control. There was no 
significant difference in the immune organ indexes of thymus, bursa and spleen (control group) as compared to the groups, only a slight decrease in chicken treated with 200 and $400 \mathrm{mg} / \mathrm{kg}$ of AMX and PMX (Figure 2I). The expression levels of IgG and IgA in the 200 group were remarkable lower $(30 \%, 10 \%)$ than those in the control group, (Figure 2J-K).

Oxidative stress indicators (AST, ALT, T-SOD, MDA and CAT), which indicated tissue impairment caused by stress, toxicity and liver damage produced by antibiotics, revealed no significant effects occurring $[25,43$, 44]. The biochemical results corroborated the demonstration to the histopathological finding of both liver and adipose tissue, which further showed that an increase in mast cells in visceral fat obesity was accompanied with antibiotics [45]. Antibiotic-treated mice were decreased liver/body weight, which is similar with ours [46]. Induction and maintenance of an appropriate level of immunological activity and inflammation markers is crucial for healthy broiler growth in poultry farms [47]. Serum IgG and intestinal secretory IgA were compared between groups (Serum IgG reflected the immune state of the system, and intestinal secretory IgA reflected the immune state of the system and the intestine, respectively) [48]. The $A M X+P M X$ decreased colon mucosal secretory $\lg A(27.5 \%-28.6 \%)$ and serum $\lg G(18.2 \%-32.7 \%)$ concentration, which is similar with the chlortetracycline and salinomycin effect on IgA and serum IgG (15.7\%)[40]. Moreover, the immune organ indices of the thymus gland and bursa indicated a weaken immunity, which was decreased $12.5 \%$ and $5.3 \%$ in 200 group and $-0.57 \%$ and $4.8 \%$ in the 400 group. For example, salmonid specific and nonspecific immune responses were also depressed by the administration of tetracycline at $10 \mathrm{mg} / \mathrm{kg}$ diet [49]. It indicated that under antibiotics, which is perhaps via reducing the nutrient consumption required for maintaining immunological activity $[50,51]$.

\subsection{Antibiotics decreased the number and changed the structure of the intestinal microflora}

The fecal flora of chicken was detected in 1, 9, 16 and 23 days (Figure 3A-D). The result showed that with the increase of antibiotic concentration, the number of gut microbiota formed a concentration gradient, and it researched the lowest level on the 400 and $800 \mathrm{mg} / \mathrm{kg}$. Simultaneously, qRT-PCR quantitation of chickens' colonic microflora on the $35_{\text {nd }}$ day showed that the content of gut microbiome was declined with the increase of antibiotic concentration, and the change was similar with fecal flora (Figure 3E). 16S rRNA gene sequencing was performed using the chicken feces collected after 35 days of antibiotic treatment. By significance tests for differences in a diversity, the gut microbiota of antibiotic mice featured an increase in Shannon index (Figure 3F). This may be due to the antibacterial effect of antibiotics, which promotes the reproduction of some nonmajor bacteria in the intestinal tract. PCoA showed that with the increase of antibiotic concentration, the distance between the intestinal flora distribution and the control group increased gradually (Figure 3G).

The antibiotic-induced difference in microbiota composition was illustrated in Figure 3. Firmicutes, Proteobacteria and Bacteroidetes species were dominant in treatment groups, as was the microbiome from the control group. It had far fewer sequences from the Firmicutes and Bacteroidetes phyla, and the microbiome of antibiotic chicken had a compositional shift to Proteobacteria (Figure 3I). The ratio of Firmicutes to Bacteroidetes (Firm/Bac ratio) was decreased upon antibiotic exposure (Figure $3 \mathrm{H}$ ). A few 
bacterial genera have been identified that were significantly different in between antibiotic and control groups (Figures 3J). Lactobacillus and Bifidobacterium are widely approved probiotic genera with extensive health-promoting and immunomodulatory properties[52, 53]. Lactobacillus, Enterococcus and Romboutsia showed lower community than those in the control group (20\%), while the contents of Bacteroides was significantly higher in the group treatment with $800 \mathrm{mg} / \mathrm{kg}$ of AMX+PMX as compared to that in the control group (354.67\%). Additionally, few other genera including Lachnospiraceae, Clostridiales, Oscillibacter and Roseburia were altered by calorie absorption. Together, these data suggested that the total and architecture of gut microbiota had a slight altered by antibiotic intervention, likely contributing to the metabolic benefits of calorie absorption.

The chicken gastrointestinal tract is home to an ecosystem rich in microbial biodiversity, playing home to $\geq 500$ phylotypes or $\sim 1$ million bacterial genes, which is integral in multiple physiological processes of the host, including being a key factor involved in host metabolism, body weight and energy homeostasis $[54,55]$. Symbiotic microbes that receive their nutrition from animal and, in turn, contribute essential nutrients and play a role in immune defense [56]. Increasing evidence showed that the nutritional value of food is influenced by the structure and operation of the gut microbial community [57]. Firmicutes (Gram positive), Bacteroidetes (Gram negative), and Actinobacteria (Gram positive) represent over $90 \%$ of the phyla and dominate the gut microbiota [58]. The alteration of the proportion of Firmicutes and Bacteroidetes changes the nutrient load in the gastrointestinal tract [59]. For example, vancomycin treatment reduced the relative abundance of Firmicutes from $37 \%$ to $50 \%$ and increased in the relative abundance of Proteobacteria $₫$ which is consistent with our previous results [60]. A research found that there is a positive correlation between ratios of Bacteroidetes to Firmicutes and the plasma glucose concentration [61]. The conversation of Firmicutes and Proteobacteria phyla was decreased 29.5\%-83.4\%, which accelerate catalyze the conversion of choline and the progression of T2D [62]. Additionally, antibiotic such as avilamycin, reduced overall community diversity and certain bacterial species (Lactobacillus and Bifidobacterium) and predominantly alter the ileal microbiota (lactobacilli dominant) $[63,64]$. The growth of Bifidobacterium is able to increase probiotic genera such as Kurthia, Lactobacillus and Bifdobacterium and meanwhile decrease harmful bacteria such as Pseudomonas (Supplemental figure 2). Lactobacillus species in the colon is crucial to produce specific fermentation products (i.e., short-chain fatty acids, predominantly acetate, propionate and butyrate) to mediate the host metabolic health [65].

\subsection{The role of antibiotic and metabolomic correlation of intestinal microbiota}

The fecal metabolite levels in chicken administered with different concentrations of AMX+PMX or were untreated (control) have been investigated. About 14,957 metabolic features was observed after untargeted LC-MS analysis and 5,861 features were annotated by following the standard filter criteria (features detected in $<70 \%$ of the QC samples were removed). PCA and PLS-DA indicated that the antibiotic treatment group and the control group could be separated with the increase of antibiotic concentration, and the metabolites of the group with $800 \mathrm{mg} / \mathrm{kg}$ of $A M X+P M X$ and the control group were significantly different (Figure 4A, B). Proteobacteria, Actinobacteria, Firmicutes, and Acidobacteria were 
primary bacteria of gut microbioma. Regulatory 44 metabolisms were classified to phylum, among them 10 were related to Deinococcus-Thermus, 1 was related to Bacteroidetes, 1 was related to Actinobacteria, and 32 were related to Opisthokonta (Figure 5, Supplemental file 2).

The gastrointestinal tract represents the interface between ingested nutrients and the host where energy is effectively extracted [66]. In healthy individuals, indigestible carbohydrates and proteins that enter the colon represent between $10 \%$ and $30 \%$ of total ingested energy and were digested by colonic microbiota[66, 67]. Meanwhile, it could be expected to contribute anywhere from $6 \%$ to $22 \%$ of daily caloric turnover[68]. A large remove of colonic microbiota can bring down the metabolic rate and loss of the "metabolic buffering" function of the gut microbiota, leading to abrogation of CR-mediated body weight loss [69]. Proteobacteria and Actinobacteria were abundant in liver injury animals[70]. Bacteroidetes are among the major members of the microbiota of animals, which are increasingly regarded as specialists for the degradation of high molecular weight organic matter, proteins and carbohydrates [71]. Some Lactobacilli possessing lipolytic activities produce significant amounts of fatty acids with antimicrobial potential under specific conditions[72, 73]. Hence, the effect of antibiotics on the intestinal flora structure and quantity may change the produces and releases an enormous array of compounds which may further act upon host tissues modulating appetite, gut motility, energy uptake and storage, and energy expenditure.

\subsection{Antibiotic treatment enhanced lipid and amino acid metabolism pathways of gut microbiome associated with obesity}

Compared with the control group, 794 differential metabolites were screened in the antibiotic groups, of which 235 metabolites were significantly downregulated $(P<0.05)$, and 559 metabolites were significantly upregulated $(P<0.05)$ (Supplemental figure). According to the material information, significant differences have been observed in the top 10 pathways of different metabolites using the KEGG $(P<0.05)$ (Figure $4 C)$. The top 5 as regards impact are tryptophan metabolism, $A B C$ transporters, aminoacyl-tRNA biosynthesis, vitamin digestion and absorption, protein digestion and absorption and those linked to the immune system. Some unique metabolites, 7 amino acids (D L-(-)-Threonine, LPhenylalanine, DL-Lysine, L-Isoleucine, Valine, Amidinoproline and Hydroxylysine), 3 steroids (tyramine sulfate and 3-(2-Acetamidoethyl)-1 $\mathrm{H}$-indol-5-yl hydrogen sulfate), 3 hormones (adrenaline, hexoprenaline and kinetin) and some metabolites, including 5-Hydroxy-DL-tryptophan, Fructoselysine, N6-Capryloyl lysine, dihydroxyphenylalanine, indole, Dimethyl 22'-azobis(2-methylpropionate), threonylphenylalanine, carbobenzoxyglycylphenylalanine, 2-methylhippuric Acid and methylhippuric acid overlapped among 5 groups $(p<0.05)$. Most of amino acids were increased in the antibiotic groups, including DL-(-)-Threonine (57.7\%-141.0\%), L-Phenylalanine (264.3\%-149.1\%), 5-Hydroxy-D L-tryptophan (196.8\%-269.7\%), Fructoselysine (250.3\%-519.7\%), DL-Lysine (49.8\%-187.9\%), L-Isoleucine (24.3\%-64.7\%). Most of them increased in the 400 group, but came close to the control group which were treated with $800 \mathrm{mg} / \mathrm{kg}$ (Figure 6). 
In serum metabolism, the differential metabolites were screened using hierarchical cluster analysis (Supplemental figure 5). The KEGG enrichment analysis of the differential metabolites showed that the influence was related to the biosynthesis of unsaturated fatty acids, glycerophospholipid metabolism, retinol metabolism pathways and choline. Moreover, products including 1-arachidonoyl-sn-glycero-3phosphocholine, 19-hydroxycholest-5-en-3-yl acetate, Vitamin A, were significantly decreased in the 400 group (Figure 7). The lipidomic further verified the effects of antibiotics in lipid metabolism. The key metabolism productions of these pathways, such as Lipolysis, TAG and cholecalciferol (Vitamin D) were changed in antibiotic group, especially in 400 group. Specific metabolites related to lipid metabolism increased when antibiotic intervention, including palmitoylcarnitine and octyl phosphate. The insulin resistance pathway was also regulated by antibiotic intervention (Supplemental Figure 6). Besides, antibiotics induced a lower fasting blood glucose level compared to that in the control group with a 16-h fast blood glucose level. However, there is a significant difference was found between fasting and fed glucose in 200 and 400 groups an hour after eating (Figure 7E, F).

The microorganisms in the gastrointestinal tract play a significant role in nutrient uptake, vitamin synthesis, energy harvest, inflammatory modulation, and host immune response [74]. For example, the weight gain occurs even when energy intake decreasd by $30 \%$ compared to common mice which remained germ-free [75]. Dysregulated fatty acid and amino acid metabolism are reported in adults with type 2 diabetes [76]. Hydroxytryptophan can help the body balance on weight, which is at a low level in obesity $[77,78]$. Diabetic rats increased the content of phenylalanine $(65.6 \%)$, valine $(29.8 \%)$, methionine (29.6\%) and aspartic acid (44.6\%), but tyrosine (48.6\%), alanine (16.8\%), lysine (13.2\%), threonine (41.4\%) and histidine (19.1\%) content were reduced, which is similar with our research [79]. Tryptophan / kynurenine which could confer benefits of feed utilization, body composition and antioxidative capacity, showed an upward trend (26.6\%) in weight in 200 group. However, it began to decrease $(64.4 \%)$ when the concentration of AMX+PMX reached $400 \mathrm{mg} / \mathrm{kg}$ [80]. It showed that amino acid levels with a significant change in body adiposity, which may be due to increased utilization of host or decreased production of amino acids by bacteria [81]. Meanwhile, some metabolites associated with the digestion and absorption of nutrients have similar response with obesity. Lysolecithin could confer benefits of feed utilization, body composition and antioxidative capacity of the channel [80]. Oxalosuccinic acid and phosphatidylcholine were reduced in serum levels of diabetes [82]. Decreased levels of 2-methylhippuric Acid and methylhippuric acid have also been related to obesity [83]. Acetyl-L-carnitine, as a long-chain fatty acid derivative, reversed the inhibition mediated by carnitine to slow the transport of fatty acids into the mitochondrial matrix where they are used for energy production [84].

Another feature of the dysbiosis in the microbiota metabolism was the levels of some health-related hormones and purine metabolites. Uric acid and hypoxanthine, as the products of the microbial metabolism of xanthines to alter gut microbiota in the presence of insulin resistance, showed an increase (41.7\%-54.7\% and $22.2 \%-120.0 \%$, respectively) [85]. Adrenaline, the main effectors of the sympathetic nervous system, are thought to control adiposity and energy balance through several mechanisms. Kinetin induced mitotic divisions throughout the digestive tract. Both of them were high expressed in the 400 group $(241.9 \%, 87.5 \%)$, which is different from hexoprenaline $(16.1 \%)$, which may play a role in 
chicken weight gain. All these fluctuated were similar with the obesity, indicating that antibiotic treatment on gut microbiota largely abolishes the regulation of metabolism [86].

\subsection{Antibiotic intervention altered the immune response of chicken}

The alterations in the serum associated with immunity have been compared. In serum, phosphocholines changed significantly in the antibiotic groups (P区0.01). The results revealed an increase in phosphocholines concentration at a concentration of $200 \mathrm{mg} / \mathrm{kg}$, returning to the lowest level at 800 $\mathrm{mg} / \mathrm{kg}$ concentration, and its related derivatives (lysophosphatidylcholine and 1-arachidonoyl-sn-glycero3-phosphocholine) have also changed (Figure 7B, H). Some unsaturated fatty acids, such as 4phenylbutyric acid, docosahexaenoic acid, elaidolinolenic acid, pinolenic acid, L-pipecolic acid and ricinoleic acid, were fluctuated with the concentration of $A M X+P M X$, especially in the 800 group (Figure 8).

The immune system restricts the overgrowth of symbiotic bacteria and prevents external bacteria from entering the host internal organs[87]. Gut microbiota is necessary for the proper immunological development of the intestine and the host which is associated with proinflammatory and host immune responses that are alter the growth performance, and the change trend of metabolites is conducive to weight gain [51]. As shown in germ-free animals, which have underdeveloped mucosal immunological tissues compared to conventional animals [88]. The kynurenine pathway is the major route for tryptophan metabolism. The rate of Tryptophan/kynurenine is an important factor in the disease development, such as cardiovascular, which is significant increase with $400 \mathrm{mg} / \mathrm{kg}$ of AMX+PMX (27.5\%). Meanwhile, oxidative stress and immune activation as inflammation factors, showing that after antibiotic intervention, the immune response of chicken was inhibited. The changes in lipid metabolome found here display dissimilarities to some other systems involving viral infection and inflammation. For example, lysophosphocholines as a factor in immunoregulation, originate primarily in the liver and are released from larger lipids [89]. Betaine protects cells, proteins, and enzymes from environmental stress, which participates in the methionine cycle and is an important nutrient for the prevention of chronic diseases [90]. Sphingosine and sphinganine $\mathrm{N}$-acyltransferase plays an important role in the pathogenesis of animal diseases $\llbracket$ showed no significant difference in treatment groups and control group (Figure 8B-D) [91].

Significant amounts of fatty acids with antimicrobial potential under specific conditions were produced $[72,73]$. For example, 4-phenylbutyric acid can suppress oxidative stress by attenuating endoplasmic reticulum stress to provide renoprotection [92]. Docosahexaenoic acid is a long-chain polyunsaturated fatty acid that worked as a ligand for the retinoid $X$ receptor in brain [93]. Pinolenic acid, a naturallyoccurring polyunsaturated fatty acid, inhibited cell metastasis by suppressing cell invasiveness and motility [94]. L-pipecolic acid was accumulated by Escherichia coli cells and protected them while growing at inhibitory osmolarity, which is able to bind the periplasmic protein, while this protein was necessary for their uptake [95]. Ricinoleic acid is the compound of antineoplastic and immunomodulatory characteristics [96]. With the increase of antibiotics, the content of all these unsaturated fatty acids 
showed a downward trend, which is coincident with the decrease of acidogenic bacteria (Lactobacilli et a), as shown in obesity [97]. Antibiotics restrain gut microbiota, resulting in reduced selective pressure on the host's immune system, thus leading to the excessive growth of chickens[87]. Therefore, in the present study, the weight alteration might be due to the changes in the intestinal community and impaired host immunity.

Childhood adiposity has increased significantly over the past several decades, which has become a major challenge to public health worldwide[98]. An obvious relationship was found between antibiotic exposure in early life with a $6 \%$ increment in the risk of obesity[46]. The growth of broiler growth with the early intervention of antibiotics is the combination of the following three effects here (suppressing the growth of gut bacteria, inhibiting host immune response and regulation the metabolic pathway of intestinal flora): (i) suppressing the growth of indigenous gut bacteria and reducing its energy consumption, which results in more nutrients for chicken for greater weight gain [99], (ii) inhibiting the health-beneficial effect of the gut microbiota and host immune response by reducing the content of immunoglobulin and inflammation related factors $[100,101]$, and (iii) regulation of the metabolic pathway of intestinal flora including lipid, amino acid metabolism and immunity [25]. The intestinal flora is likely to have a significant impact on host physiological processes with early intervention of antibiotics, which may be similar to the factors of early childhood obesity, and it can provide a theoretical reference for the early obesity exploration of humans.

\section{Conclusion:}

Antibiotics as feed additive in the poultry industry play a key role by mediating gut microbiota in calorie absorption. The maturation of intestinal microbiota was significantly retarded and eventually delayed by different concentrations of antibiotics, with a high level of growth performance and low level of immunity response. Meanwhile, the beneficial factors produced by gut microbiota are relatively less, showing that a moderate mature gut microbiota is more inclined to increase the weight of chickens. Gut microbiota has been suggested to serve as a target in the prevention and treatment of metabolic disorders.

\section{Materials And Methods}

\subsection{Animal experiment design}

The white-feather broilers were purchased from a poultry farm in Yuzhong County, Lanzhou. A total of 84 broilers ( 1 day old) with similar weight and healthy feathers were randomly split into 7 groups (6 chickens per cage). The polymyxin and amoxicillin $(0,20,50,100,200,400,800 \mathrm{mg} / \mathrm{kg})$ were selected according to the pretreatment experiment (Supplementary file 1). The standard chicken feed was bought from Zhengda Co., LTD (Lanzhou, China). For the experimental procedures, six chickens in a cage housed at $22^{\circ} \mathrm{C} \pm 1^{\circ} \mathrm{C}$ and $60-75 \%$ relative humidity. The care and use of laboratory animals in our study was approved by Lanzhou University Animal Ethics Committee, and all the relevant ethical regulations have been followed. All broiler chickens in each group were weighed individually at each week during the full 
trial. The feed consumed for one group was monitored on a day basis. During the $35 \mathrm{nd}$ day of treatment, all chickens in each group were moved into a clean and empty cage every 7 days for an hour, and fecal samples were collected. Blood was collected from the vein and was left undisturbed for $30 \mathrm{~min}$. The collected blood samples were centrifuged at 4,000 rpm for 20 minutes to get the serum. Later on, the chicken was anesthetized by water flooding. Also, liver, adipose and intestine samples were gathered for all the respective groups. The collected samples were washed with saline, and were then collected in preweighted tubes with $1 \mathrm{~mL}$ phosphate buffer saline (PBS) diluent and were stored at $-20^{\circ} \mathrm{C}$ for future use. All samples were preserved at $-80^{\circ} \mathrm{C}$ until analysis, except those used for histopathology, which were stored in $4 \%$ paraformaldehyde.

\subsection{Tissue collection and biomarker measurements}

The liver and adipose tissue were rinsed with PBS with a volume of $0.8 \mathrm{~cm} \times 0.8 \mathrm{~cm}$. Samples were fixed with $4 \%$ paraformaldehyde solution at room temperature for more than 1 day. Later, the samples were sent to Huapu Biotechnology Co., Ltd. (Lanzhou) for immunohistochemistry analysis. Intestinal content $(15 \mathrm{ml})$ was collected and mixed with an equal volume of PBS $(\mathrm{pH} \mathrm{7.14)}$ and was centrifuged at $800 \mathrm{~g}$ for $15 \mathrm{~min}$. The supernatant was used for SIgA (ELISA). Serum total IgG was detected using enzyme-linked immunosorbent assay (ELISA). Their concentrations were then calculated from the standard curves. The thymus gland (on the right side), bursal and spleen tissues were collected simultaneously and weighed for each chicken. The immune organ index $(\mathrm{g} / 100 \mathrm{~g})$ was calculated for each of the organs as WO/WB $\times$ 100 , with WO being weight of the immune organ and WB weight of the chicken [40].

To determine the effects of antibiotics on the oxidative stress system, fresh liver and blood were taken on the $35_{\text {nd }}$ day. At first, liver samples were grinded using a handheld grinder (Jingxin, Shanghai, China) on ice. The suspension of samples was obtained with low-speed centrifugation (8000 rpm, $10 \mathrm{~min})$. The levels of the total protein, malondialdehyde (MDA), catalase (CAT), and total superoxide dismutase (TSOD) in serum samples and the ALT and AST levels in livers were all detected using commercial kits (Jiancheng, Nanjing, China) according to the protocols provided and the results were calculated according to the curve of different indexes [102].

\subsection{DNA extraction and qPCR}

In each group, fecal samples were randomly collected from the different group chicks with six copies on day 1 , day 9 , day 16 , and day 23 respectively. After that, fecal samples were collected aseptically and stored quickly on ice, then transferred to $-80^{\circ} \mathrm{C}$. The DNA from feces and colon content samples were extracted according to the manufacturer's instruction using TIANamp Stool DNA Kit (Tiangen, China). To quantify the total bacteria in the samples, primers tot- $F$ and tot- $R$ (F-tot: GCAGGCCTAACACATGCAAGTC; R-tot: CTGCTGCCTCCCGTAGGAGT) were used to amplify the 16S rDNA [103]. qPCR was run on a BioRAD CFX96 (USA). It was performed in a total volume of $10 \mu \mathrm{L}$ using SYBR Premix Ex Taq II (Takara) on real-time quantification PCR instrument (Bio-RAD CFX96, USA). All measurements were in triplicates. Standard curves were constructed using PCR product of the 16S rRNA gene of $E$. coli. The obtained PCR products were cloned into $\mathrm{T}$ vector (Takara, Dalian, China) and were transferred into $\mathrm{DH}-5 \mathrm{a}$, which was 
then incubated at $37^{\circ} \mathrm{C}$ for $6 \mathrm{~h}$ (100-140rpm) using Plasmid Mini Kit I (E.Z.N.A., Omega, USA) as the standard sample. The recombinant plasmids were used for qPCR, with $10^{3}, 10^{4}, 10^{5}, 10^{6}, 10^{7}, 10^{8}, 10^{9}$, and $10^{10}$ copies of the plasmids per reaction, which were used for calibration. The target copy numbers $(T)$ were estimated by the equation: $T=(D /(P L \times 660)) \times 6.022 \times 1,023,133$, where $D(g / l)$ and $P L$ (inbase pairs) were the plasmid DNA concentration and length, respectively. Each standard curve was generated from at least five tenfold plasmid dilutions in triplicate. The content of the whole bacterial community in different samples were calculated based on the standard curve [104, 105].

\subsection{S rRNA gene sequencing analysis}

DNA extraction from the $35_{n d}$ broiler fecal samples were examined using $1 \%$ agarose gel electrophoresis. All samples were quantified on a Qubit 2.0 Fluorometer (Thermo Fisher Scientific, USA). Then, the V3-V4 hypervariable region of the $16 \mathrm{~S}$ rRNA gene was amplified using PCR, which used 338F forward primer (5'ACTCCTACGGGAGGCAGCA-3') and the 806R reverse primer (5'- GGACTACHVGGGTWTCTAAT-3'). The PCR cycle was denatured at $94^{\circ} \mathrm{C}$ for $3 \mathrm{~min}(1 \mathrm{cycle})$; followed by $94^{\circ} \mathrm{C}$ for $45 \mathrm{~s}$, annealed at $50^{\circ} \mathrm{C}$ for $60 \mathrm{~s}$, and extended at $72^{\circ} \mathrm{C}$ for $90 \mathrm{~s}$ ( 25 cycles), with the final extension step of $72^{\circ} \mathrm{C}$ for $10 \mathrm{~min}$. The amplicon products were purified using AMPure XP beads (Beckman Coulter, USA). Sequencing libraries were generated using the TruSeq ${ }^{\circledR}$ DNA PCR-Free Sample Preparation Kit (Illumina, USA) following the manufacturer's recommendations. Then library quality was assessed using the Agilent Bioanalyzer 2100 system (Agilent Technologies, USA). The quality libraries were finally sequenced on an Illumina HiSeq 2500 platform with 250 bp of paired-end reads. Sequencing libraries were generated using the TruSeq ${ }^{\circledR}$ DNA PCR-Free Sample Preparation Kit (Illumina, USA) following the manufacturer's recommendations. Then library quality was assessed using the Agilent Bioanalyzer 2100 system (Agilent Technologies, USA). The quality libraries were finally sequenced using an Illumina HiSeq 2500 platform with 250 bp of paired-end reads [106].

The raw paired-end reads were overlapped and merged to get raw tags (SUB9686300) using the FLASH software (v1.2.7) [107]. Trimmomatic (v0.33) is utilized to filter low-quality raw tags and to keep highquality clean tags. Moreover, clean tags were imported into the software package Quantitative Insights Into Microbial Ecology 2 (QIIME2 version 2018-8) [108, 109]. Taxonomy was assigned using the $99 \%$ identity SILVA (release 132) V3-V4 classifier. All the ribosomal sequence variants (RSVs) were identified as unique features across all samples without clustering. The feature table, rooted phylogenetic tree, representative sequences and metadata from QIIME2 were then exported for further analysis in $\mathrm{R}$ (V3.3.1). R package ade4 has been ordinated in explanatory matrices using principal component analysis (PCA) including infection time course as explanatory variables. Alpha and beta diversity were computed using a rarefaction depth of sequences. Differences in bacterial alpha diversity (Shannon's index, observed features and evenness index) between each study group were evaluated based on the rarefied data and were tested using the Wilcox rank-sum method. Beta diversity was calculated using the BrayCurtis distance, Jaccard distance, unweighted UniFrac distance and weighted UniFrac distance by the $R$ package VEGAN (V2.5-3), respectively [110]. Differences in beta diversity were identified using analysis of similarity (ANOSIM) and effect size indicated by an R-value (between -1 and $+\mathrm{I}$, with a value of 0 
representing the null hypothesis). Community structure difference based on beta diversity was visualized using nonmetric Multidimensional Scaling (NMDS) method by R package VEGAN. Resulting abundance tables were analyzed using the LefSe pipeline to find out representing differential bacterial taxon among multiple sample groups (Segata, et al., 2011). The threshold logarithmic linear discriminant analysis (LDA) score was set for biomarker detection of microbiota composition and community function. The tool PICRUSt (Langille et al., 2013) was applied to predict potential functional changes of the microbiome by inferring the metagenomes from $16 \mathrm{~S}$ sequences [111].

\subsection{Feces and serum samples preparation for metabonomic analysis}

Fecal samples were stored at $-80^{\circ} \mathrm{C}$ until processing. Preparation was carried out as described previously at Metabolon, Inc [112]. Lyophilized fecal samples were extracted at a constant per-mass basis. To suspend $1 \mathrm{~g}$ feces, $1 \mathrm{ml}$ of $\mathrm{ddH}_{2} \mathrm{O}$ was suspend centrifuged for 10 min with $3,000 \times \mathrm{g}$, and the supernatant was removed. Moreover, $500 \mu \mathrm{L} \mathrm{ddH_{2 }} \mathrm{O}$ was added into the precipitate again, and the feces were suspended again, and was centrifuged for 5 min with 10,000 g. The supernatant was taken and combined. Aacetonitrile was added to the final concentration of $1 \%$, while formic acid was added to the final concentration of $0.5 \%$. After mixing, $13,000 \mathrm{~g}$ was centrifuged for $15 \mathrm{~min}$, the supernatant was put into an ultrafiltration tube, 4,500 g was taken, and the liquid in the tube was taken after $50 \mathrm{~min}$. Then, 200 $\mu \mathrm{L}$ of each liquid was evaporated to dryness by centrifugal concentrator and stored in $-80^{\circ} \mathrm{C}$ refrigerator. After all the samples were processed, $200 \mu \mathrm{L}$ was added to each dry sample for dissolution and was placed at $4^{\circ} \mathrm{C}$ for $8 \mathrm{~h}$. The supernatant was extracted and detected by LC-MS [113].

For metabolomics analysis, a volume of $50 \mathrm{~mL}$ from each serum sample was thawed at $4^{\circ} \mathrm{C}$. Serum proteins were precipitated using $200 \mu \mathrm{L}$ ice-cold methanol, which contained 9-fluorenylmethoxycarbonylglycine $(10 \mu \mathrm{g} / \mathrm{mL})$ as an internal standard. After vortexing, the mixture was centrifuged at $16,000 \mathrm{rpm}$ for $10 \mathrm{~min}$ at $4^{\circ} \mathrm{C}$, and the supernatant was divided into two parts, one for liquid chromatography/mass spectrometry (LC-MS) analysis. All samples were kept at $4^{\circ} \mathrm{C}$ and were analyzed within $48 \mathrm{~h}$. In order to prevent batch effect, the assays were conducted in a random manner.

For lipidomics analysis, lipids were extracted from the serum using a modified Bligh and Dyer method. Briefly, $900 \mu \mathrm{L}$ of chloroform-methanol, 1:2 (v/v) was added to $100 \mu \mathrm{L}$ serum. After 20 min vortexing and incubation at $4^{\circ} \mathrm{C}, 300 \mu \mathrm{L}$ of chloroform and $300 \mu \mathrm{L}$ of $\mathrm{ddH}_{2} \mathrm{O}$ were added to the mixture and were centrifuged at $9,000 \mathrm{rpm}, 4^{\circ} \mathrm{C}$ for $2 \mathrm{~min}$. Lipids were then recovered from the lower organic phase after centrifugation. Subsequently, $500 \mu \mathrm{L}$ of chloroform was added and was vortexed at $4^{\circ} \mathrm{C}$ for $20 \mathrm{~min}$. After centrifugation, lipids were recovered from the organic phase and were combined with the previous fraction. The lipid extracts were vacuum-dried, stored at $-80^{\circ} \mathrm{C}$ and analyzed within a week [114].

\subsection{Metabolomics analysis by LC-MS}

The supernatant fraction from the sample preparation step was analyzed using liquid chromatograpytandem mass spectrometry (LC-MS/MS) analysis on a Thermo Scientific Dionex UltiMate 3,000 ultrahigh 
pressure liquid chromatography system (Thermofisher, USA) equipped with a Thermo Orbitrap Fusion Lumos mass spectrometer detector managed using a Thermo Xcalibur workstation. The column used for the separation was a Thermo Scientific ${ }^{\text {TM }}$ Hypersil GOLD ${ }^{\text {TM }}$ C18 $\left(2.1 \mathrm{~mm}, 1.9\right.$ um; Thermo Scientific ${ }^{\text {TM }}$; USA). The oven temperature was set at $35^{\circ} \mathrm{C}$. The gradient elution involved a mobile phase consisting of $0.1 \%$ formic acid in water and methanol. The initial condition was set at $2 \% \mathrm{~B}$. A linear gradient for 30 min was applied to $30 \%$ B, followed by 8 min gradient to $60 \%$ B and then by a 2 min gradient to $100 \%$ and held 5 $\mathrm{min}$. then returned to starting conditions over $1 \mathrm{~min}$. Flow rate was set at $0.3 \mathrm{ml} / \mathrm{min}$, and $8 \mathrm{uL}$ of samples was injected. The electrospray ionization mass spectra were acquired in positive ion mode. Mass data were collected between 100 and $1,000 \mathrm{~m} / \mathrm{z}$ at a rate of two scans per second. The ion spray voltage was set at $3,500 \mathrm{~V}$, the ion transfer tube temperature was maintained at $320^{\circ} \mathrm{C}$, and the vaporize temperature is $400^{\circ} \mathrm{C}$. The instrument method consisted of one full MS scan from $100-1000 \mathrm{~m} / \mathrm{z}$ with orbitrap detector, the detector resolution is 120,000 and the maximun injection time is $50 \mathrm{~ms}$. The sheath gas and aux gas flow rates were 40 Arb and 50 Arb, respectively. The MS2 scan range mode is Auto : m/z Normal with Orbitrap detector, first mass is $50 \mathrm{~m} / \mathrm{z}$; and the resolution is 15000 .

The stability of the LC-MS method was examined and evaluated using a subset of peaks covering a range of masses, intensities, and retention times across the QC samples. The original total ion current (TIC) chromatogram of the quality control samples was observed, the retention time reproducibility of the preliminary analysis instrument and the number of peaks measured were analyzed, and the detection situation of the compounds was judged. The reproducibility of the internal standard for quality control of data was analyzed by using a blank sample, one-dimensional PCA diagram of QC sample, and a correlation of QC sample, which showed that the system was stable $[114,115]$.

\subsection{Data analysis}

All data were recorded using the Xcalibur software and were exported as a raw format for further analysis. Mass spectra were digitally analyzed using the Compounds discoverer 3.2 (Thermo Fisher Ltd, USA). The statistical calculation was performed using the maximum intensity of the metabolite ion

peaks. The date was combined into a single matrix by aligning peaks with the same mass-retention time paired together from each date file in the data set. The intensities for each peak were normalized to the sum of the peak intensity for each data set [116]. To support the metabolite identification, the following databases have been used: KEGG (http://www.kegg.com/), LipidMAPS database (http://www.lipidmaps.org/data/strycture/), PubChem compound database (http://ncbi.nim.nih.gov/) and the human metabolite data base (http://www.hmdb.ca/) [117].

\subsection{Compound identification and pathways analysis}

The original data were analyzed by R Software (V3.5.1), including PCA of unsupervised pattern and orthogonal partial least squares discriminant analysis (OPLS-DA) of supervised pattern, respectively. The interpretation rate of experimental interventions (principal components) or models was obtained, and the modeling effect of antibiotics exposure in chicken was evaluated. Compared with PCA, OPLS-DA can use the classification information of samples for modeling, so as to enlarge the differences between groups, 
eliminate the variables irrelevant to classification, reduce noise interference, highlight important variables, and facilitate the identification of marker metabolites. The PLS loading plot was used to identify metabolites with major influence on the group membership. All the compounds detected in the all groups were screened using the KEGG database, and the screening criteria were set to meet the following three conditions: (1) the contribution value of different metabolites to this group was greater than $1 ;(2)$ the similarity with the corresponding substances in KEGG database was greater than 1 ; (3) a significant difference in the content of metabolites was found between the night light exposure group and the control group $(P<0.05)$. The selected differential metabolites were introduced into analyst

(https://www.metaboanalyst.ca.). The path analysis module in the software automatically generates metabolic pathway map after matching with the name of metabolite in the KEGG database[117, 118].

\subsection{Co-occurrence network analysis}

The R package was used for calculating the Poisson correlation coefficient of metabolites and community. If $\mathrm{P}<0.05$ and the absolute value of correlation coefficient $>0.4$, it is considered that there is correlation. The correlation relationship after filtering was obtained, and r-packet iGraph was used to plot. In each group, $16 \mathrm{~S}$ amplicon sequencing and metabolomic data on day 35 was first pooled together to create the global network patterns, and then separately analyzed to illustrate the change of network density and network centralization.

\subsection{Statistical analysis}

Statistical analysis was performed using the SPSS 17.0 software. A P-value of $<0.05$ was regarded as a significant difference criterion. Duncan's multiple range tests for statistical differences at a confidence level of 95\% $(p<0.05)$ was applied. Graph-pad Prism 8.0 and Origin 8.0 were used as graphics-rendering software.

\section{Abbreviations}

ADG Average daily gain

ANOSIM Analysis of similarity

LDA Linear discriminant analysis

NMDS Nonmetric multidimensional scaling

PBS Phosphate buffer saline

PCA Principal component analysis

PCoA Principal coordinate analysis

RSV Ribosomal sequence variants 
TIC Total ion current

\section{Declarations}

\section{Acknowledgment}

We thank all participants of the study and the Central Lab of School of Life Sciences, Lanzhou University for providing all necessary equipment.

\section{Availability of data and materials}

Sequence data used in the study is available at National Center for Biotechnology Information Search database (SUB9686300).

\section{Authors' contributions}

Ying Wu designed the experiment, analyzed the data and wrote the manuscript. Liang Peng guided the bioinformatic analyses and data curation. Rong Han, Pengya Feng and Aman Khan provided advice and review this manuscript. Pu Liu and Xiangkai Li provided advice, supervision and revised this manuscript. All authors read and approved the final manuscript.

\section{Funding}

The work was supported by Gansu Province Major Science and Technology Project (Grant No:

17ZD2WA017), National Natural Science Foundation of China (Grant No: 31870082) and Fundamental Research Funds for the Central Universities (Grant No: Izujbky-2020-83).

\section{Ethics approval and consent to participate}

Not applicable.

\section{Consent for publication}

Not applicable.

\section{Competing interests}

The authors declare that they have no known competing financial interests or personal relationships that could have appeared to influence the work reported in this paper. Author declare no conflict of interest.

\section{Author details}

${ }^{1}$ Gansu Key Laboratory of Biomonitoring and Bioremediation for Environmental Pollution, School of Life Sciences, Lanzhou University, Lanzhou, China. 
${ }^{2}$ Ministry of Education Key Laboratory of Cell Activities and Stress Adaptations, School of Life Science, Lanzhou University, Lanzhou, China.

\section{References}

1. Manafi M. Comparison Study of a Natural Non-Antibiotic Growth Promoter and a Commercial Probiotic on Growth Performance, Immune Response and Biochemical Parameters of Broiler Chicks. Journal of Poultry Science 2015;52:S27.

2. Pagel SW, Gautier P. Use of antimicrobial agents in livestock. Revue Scientifique Et Technique 2012;31:145.

3. Tiseo K, Huber L, Gilbert M, Robinson TP, Boeckel TPV. Global Trends in Antimicrobial Use in Food Animals from 2017 to 2030. Antibiotics 2020;9:918.

4. Reducing antimicrobial use in food animals. Science 2017;357:1350-2.

5. Bai Y, Xu R, Wang QP, Zhang YR, Yang ZH. Sludge anaerobic digestion with high concentrations of tetracyclines and sulfonamides: Dynamics of microbial communities and change of antibiotic resistance genes. Bioresource technology 2019;276:51-9.

6. Su S, Zhang M, Li B, Zhang H, Dong X. HPLC determination of sulfamethazine in milk using surfaceimprinted silica synthesized with iniferter technique. Talanta 2008;76:1141-6.

7. Cho I, Yamanishi S, Cox L, Methé BA, Zavadil J, Li K, et al. Antibiotics in early life alter the murine colonic microbiome and adiposity. Nature.

8. Ozawa E. Studies on growth promotion by antibiotics. I. Effects of chlortetracycline on growth. Journal of Antibiotics 1955;8:205.

9. Manafi M, Hedayati M, Pirany N, Akwu OA. Comparison of performance and feed digestibility of the non-antibiotic feed supplement (Novacid) and an antibiotic growth promoter in broiler chickens. Poultry Science 2018.

10. Feighner SD, Dashkevicz MP. Subtherapeutic levels of antibiotics in poultry feeds and their effects on weight gain, feed efficiency, and bacterial cholytaurine hydrolase activity. Appl. Environ. Microbiol., 53, 331-6. Applied \& Environmental Microbiology 1987;53:331-6.

11. Maron DF, Smith TJ, Nachman KE. Restrictions on antimicrobial use in food animal production: an international regulatory and economic survey. Globalization and Health 2013;9.

12. Lhermie G, Tauer LW, Grhn YT. The farm cost of decreasing antimicrobial use in dairy production. PLoS ONE 2018;13:e0194832.

13. Martínez-Carballo E, González-Barreiro C, Scharf S, Gans O. Environmental monitoring study of selected veterinary antibiotics in animal manure and soils in Austria. Environmental Pollution 2007;148:570-9.

14. Wong CB, Odamaki T, Xiao J-z. Beneficial effects of Bifidobacterium longum subsp. longum BB536 on human health: Modulation of gut microbiome as the principal action. Journal of functional foods 2019;54:506-19. 
15. Yamanaka M, Nomura T, Kametaka M. Role of intestinal microbes in body composition in germ-free, gnotobiotic and conventional mice. Journal of Nutritional Science \& Vitaminology 1977;23:211.

16. Mahana D, Trent CM, Kurtz ZD, Bokulich NA, Battaglia T, Chung J, et al. Antibiotic perturbation of the murine gut microbiome enhances the adiposity, insulin resistance, and liver disease associated with high-fat diet. Genome Medicine 2016;8:48.

17. Hedde RD, Lindsey TO. Virginiamycin: a nutritional tool for swine production. Agri-Practice (USA) $1986 ; 7: 70-2$.

18. Saunders DR, Sillery J. Effect of lactate and H+ on structure and function of rat intestine. Implications for the pathogensis of fermentative diarrhea. Digdissci 1982;27:33-41.

19. Pirofski LA, Casadevall A. The Damage-Response Framework of Microbial Pathogenesis and Infectious Diseases. Advances in Experimental Medicine \& Biology 2008;635:135-46.

20. Bremener SM, Alferova VA, Zgurskaja GN, Zubkova El, Pipko AS, Rogova KP. Changes in the metabolism of vitamins B6 and B12, nicotinic acid, pantothenic acid and vitamin $C$ in patients with chronic colitis of different causes and treated with tetracycline. Antibiotiki 1967.

21. Selwyn FP, Csanaky IL, Zhang Y, Klaassen CD. Importance of Large Intestine in Regulating Bile Acids and GLP-1 in Germ-free Mice. Drug metabolism and disposition: the biological fate of chemicals 2015;43:1544.

22. Theriot CM, Koenigsknecht MJ, Carlson Jr PE, Hatton GE, Nelson AM, Li B, et al. Antibiotic-induced shifts in the mouse gut microbiome and metabolome increase susceptibility to Clostridium difficile infection. Nature communications 2014;5:3114.

23. Natividad JM, Allison A, Julien P, Bruno L, Charlotte JA, Rebeca M, et al. Impaired Aryl Hydrocarbon Receptor Ligand Production by the Gut Microbiota Is a Key Factor in Metabolic Syndrome. Cell Metabolism 2018;28:S1550413118304443-.

24. Choi HK, Atkinson K, Karlson EW, Willett W, Curhan G. Purine-rich foods, dairy and protein intake, and the risk of gout in men.

25. Zarrinpar A, Chaix A, Xu ZZ, Chang MW, Marotz CA, Saghatelian A, et al. Antibiotic-induced microbiome depletion alters metabolic homeostasis by affecting gut signaling and colonic metabolism. Nature communications 2018;9:2872.

26. Pérez-Cobas AE, Gosalbes MJ, Friedrichs A, Knecht H, Artacho A, Eismann K, et al. Gut microbiota disturbance during antibiotic therapy: a multi-omic approach. Gut Microbes 2013;5:64-70.

27. Morgun A, Dzutsev A, Dong X, Greer RL, Sexton DJ, Ravel J, et al. Uncovering effects of antibiotics on the host and microbiota using transkingdom gene networks. Gut 2015:1732-43.

28. Yerevanian A, Soukas AA. Metformin: Mechanisms in Human Obesity and Weight Loss. Current Obesity Reports 2019.

29. Kelly CJ, Colgan SP, Frank DN. Of microbes and meals: the health consequences of dietary endotoxemia. Nutrition in Clinical Practice 2012;27:215-25. 
30. Vrieze A, Out C, Fuentes S, Jonker L, Reuling I, Kootte RS, et al. Impact of oral vancomycin on gut microbiota, bile acid metabolism, and insulin sensitivity. Journal of Hepatology 2014.

31. Hu X, Wang T, Liang S, Li W, Wu X, Jin F. Antibiotic-induced imbalances in gut microbiota aggravates cholesterol accumulation and liver injuries in rats fed a high-cholesterol diet. Applied Microbiology\&Biotechnology 2015.

32. Pupavac S, Trifunovic' M, Sinovec Z. Use of antibiotics and probiotics for stimulative purposes in pig diet. Veterinarski Glasnik 2000.

33. Pelicano E, Souza P, Souza H, Figueiredo D, Boiago M, Carvalho S, et al. Intestinal mucosa development in broiler chickens fed natural growth promoters. Revista Brasileira De Ciência Avícola 2005;7:221-9.

34. Shuo W, Meiqin $H$, Xue Y, Jingyu Z, Lanlan C, Lin W, et al. Gut microbiota mediates the anti-obesity effect of calorie restriction in mice. Scientific Reports 2018;8:13037-.

35. Zhu YG, Johnson TA, Su JQ, Qiao M, Guo GX, Stedtfeld RD, et al. Diverse and abundant antibiotic resistance genes in Chinese swine farms. Pnas 2013;110:3435-40.

36. Roth FX, Gotterbarm GG, Windisch W, Kirchgessner M. Whole-body protein turnover and nitrogen balance in growing pigs supplied with an antibiotic feed additive (Avilamycin). Journal of Animal Physiology \& Animal Nutrition 2010;82:88-93.

37. Le T, Sun Q, Xie Y, Shu L, Cao X. A Highly Sensitive Aptasensor for Sulfamethazine Detection Using an Enzyme-Linked Aptamer Assay. Food Analytical Methods 2018;11.

38. Broom, Leon J. The sub-inhibitory theory for antibiotic growth promoters. Poult 2017.

39. Barton MD. Antibiotic use in animal feed and its impact on human healt. Nutrition research reviews 2000;13:279-99.

40. Gao P, Ma C, Sun Z, Wang L, Huang S, Su X, et al. Feed-additive probiotics accelerate yet antibiotics delay intestinal microbiota maturation in broiler chicken. Microbiome 2017;5:91.

41. Reda RM, Ibrahim RE, Ahmed E, El-Bouhy ZM. Effect of oxytetracycline and florfenicol as growth promoters on the health status of cultured Oreochromis niloticus. Egyptian Journal of Aquatic Research 2013;39:241-8.

42. Jukes HG, Hill DC, Branion HD. Effect of Feeding Antibiotics on the Intestinal Tract of the Chick. Poultry Science 1956;35:716-23.

43. Rouf MA, Das AK, Mitu NL, Shahriar M, Mitu L. Evaluation of hepatoprotective effects of arogyavardhini against D-galactosamine-induced hepatotoxicity in rats. Journal of Pharmacognosy and Phytochemistry 2021;10:12-9.

44. Brandtzaeg P. Role of secretory antibodies in the defence against infections. International Journal of Medical Microbiology 2003;293:3-15.

45. Altintas MM, Azad A, Nayer B, Contreras G, Nayer A. Mast cells, macrophages, and crown-like structures distinguish subcutaneous from visceral fat in mice. Journal of Lipid Research 2011;52. 
46. Shao X, Ding X, Wang B, Ling L, An X, Yao Q, et al. Antibiotic Exposure in Early Life Increases Risk of Childhood Obesity: A Systematic Review and Meta-Analysis. Frontiers in Endocrinology 2017;8:170-.

47. Smit LAM, Femke SDB, Opstal-Van WAWJ, Mari?Tte H, Johan B, Wouters IM, et al. Q Fever and Pneumonia in an Area with a High Livestock Density: A Large Population-Based Study. Plos One 2012;7.

48. Pascal, Pierre, and, Agnes, Langendries, and, et al. Cholera toxin neutralization: a comparison of purified serum IgG and biliary secretory IgA antibodies - ScienceDirect. Immunology Letters 1988;18:51-5.

49. Siwicki AK, Anderson DP, Dixon OW. Comparisons of nonspecific and specific immunomodulation by oxolinic acid, oxytetracycline and levamisole in salmonids. Veterinary Immunology \& Immunopathology 1989;23:195-200.

50. Lin J, Hunkapiller AA, Layton AC, Chang YJ, Robbins KR. Response of intestinal microbiota to antibiotic growth promoters in chickens. Foodborne Pathogens \& Disease 2013;10:331-7.

51. Niewold TA. The nonantibiotic anti-inflammatory effect of antimicrobial growth promoters, the real mode of action? A hypothesis. Poultry Science 2007;86:605.

52. Delzenne, Nathalie M, Neyrinck, Audrey M, Bäckhed, Fredrik, et al. Targeting gut microbiota in obesity: effects of prebiotics and probiotics. Nature Reviews Endocrinology 2011.

53. Shinji, Fukuda, Hidehiro, Toh, Todd, D., et al. Acetate-producing bifidobacteria protect the host from enteropathogenic infection via carbohydrate transporters. Gut Microbes 2012;3:449-54.

54. Jiangrang, Lu, Umelaalim, Idris, Barry, Harmon, et al. Diversity and Succession of the Intestinal Bacterial Community of the Maturing Broiler Chicken. Applied and Environmental Microbiology 2003;69:6816-24.

55. Kogut $\mathrm{MH}$. The gut microbiota and host innate immunity: Regulators of host metabolism and metabolic diseases in poultry? Journal of Applied Poultry Research 2013;22:637-46.

56. Chow J, Mazmanian SK. A Pathobiont of the Microbiota Balances Host Colonization and Intestinal Inflammation. Cell host \& microbe 2010;7:265-76.

57. Kau AL AP, Griffin NW, Goodman AL, Gordon Jl. Human nutrition, the gut microbiome and the immune system. Nature 2011;474:327-36.

58. Ley RE, Backhed F, Turnbaugh P, Lozupone CA, Knight RD, Gordon Jl. Obesity alters gut microbial ecology. Proceedings of the National Academy of Sciences of the United States of America 2005;102:11070-5.

59. Reiner J, Duc Son L, Turnbaugh PJ, Cathy T, Clifton B, Gordon JI, et al. Energy-balance studies reveal associations between gut microbes, caloric load, and nutrient absorption in humans. The American journal of clinical nutrition 2018;2011凶94凶10:58-65区.

60. Fujisaka S, Ussar S, Clish C, Devkota S, Kahn CR. Antibiotic effects on gut microbiota and metabolism are host dependent. Journal of Clinical Investigation 2016;126:4430. 
61. Larsen N, Vogensen FK, Van dB, Frans W. J., Nielsen DS, Andreasen AS, Pedersen BK, et al. Gut Microbiota in Human Adults with Type 2 Diabetes Differs from Non-Diabetic Adults. Plos One 2010;5:e9085.

62. Guan M, Xie L, Diao C, Wang N, Hu W, Zheng Y, et al. Systemic Perturbations of Key Metabolites in Diabetic Rats During the Evolution of Diabetes Studied by Urine Metabonomics. Plos One 2013;8:132-.

63. La-Ongkhum O, Pungsungvorn N, Amornthewaphat N, Nitisinprasert S. Effect of the antibiotic avilamycin on the structure of the microbial community in the jejunal intestinal tract of broiler chickens. Poultry Science 2011;90:1532-8.

64. Luthold RV, Fernandes GR, Moraes A, Folchetti L, Ferreira S. Gut microbiota interactions with the immunomodulatory role of vitamin D in normal individuals. Metabolism: clinical and experimental 2017;69:76-86.

65. Venema K. Role of gut microbiota in the control of energy and carbohydrate metabolism. Current Opinion in Clinical Nutrition \& Metabolic Care 2010;13:432-8.

66. Krajmalnik-Brown, R., Ilhan, Z.-E., Kang, D.-W., et al. Effects of Gut Microbes on Nutrient Absorption and Energy Regulation. NUTRITION IN CLINICAL PRACTICE 2012.

67. Bergman EN. Energy contributions of volatile fatty acids from the gastrointestinal tract in various species. Physiological Reviews 1990;70:567-90.

68. Riedl RA, Atkinson SN, Burnett C, Grobe JL, Kirby JR. The Gut Microbiome, Energy Homeostasis, and Implications for Hypertension. Current Hypertension Reports 2017;19:27.

69. Lindsay EC, Metcalfe NB, Llewellyn MS. The potential role of the gut microbiota in shaping host energetics and metabolic rate. Journal of Animal Ecology 2020.

70. Kirpich IA, Petrosino J, Ajami N, Feng W, Wang Y, Liu Y, et al. Saturated and Unsaturated Dietary Fats Differentially Modulate Ethanol-Induced Changes in Gut Microbiome and Metabolome in a Mouse Model of Alcoholic Liver Disease. American Journal Of Pathology 2016;186:765-76.

71. Thomas F, Hehemann JH, Rebuffet E, Czjzek M, Michel G. Environmental and Gut Bacteroidetes: The Food Connection. Frontiers in Microbiology 2011;2:93.

72. Eagle, H. Amino Acid Metabolism in Mammalian Cell Cultures. Science 1959;130:432-7.

73. Ghosh AR. Appraisal of Probiotics and Prebiotics in Gastrointestinal Infections. 2012.

74. Mohr AE, Jger R, Carpenter KC, Kerksick CM, Antonio J. The athletic gut microbiota. Journal of the International Society of Sports Nutrition 2020;17.

75. The gut microbiota as an environmental factor that regulates fat storage. Proceedings of the National Academy of Sciences of the United States of America 2004.

76. Mihalik, S. J, Michaliszyn, S. F, DeLasHeras, J., et al. Metabolomic profiling of fatty acid and amino acid metabolism in youth with obesity and type 2 diabetes: Evidence for enhanced mitochondrial oxidation. Diabetes Care 2012. 
77. Blundell JE, Latham CJ. Sensitivity of the behavioural assay for measuring the action of drugs on feeding: effects of tryptophan and 5-hydroxy-tryptophan [proceedings]. British Journal of Pharmacology 1979;66:482P.

78. Hinz MC. Comprehensive pharmacologic therapy for treatment of obesity. US, 2002.

79. Liu JT, Xiao XJ, Lan YU, TabusiManaer, XinhuaNabi, University XM, et al. Effect of Xinjiang fermented camel milk on serum amino acid metabolism in diabetic rats. Science and Technology of Food Industry 2015.

80. Liu G, Ma S, Chen F, Gao W, Zhang W, Mai K. Effects of dietary lysolecithin on growth performance, feed utilization, intestinal morphology and metabolic responses of channel catfish (Ictalurus punctatus). Aquaculture Nutrition 2020;26.

81. Gross MM, Hecker H, Frömke C, Ayerle G, Hillemanns P. Ghrelin Treatment Increases Receptor-bound Leptin in Healthy and Endotoxemic Obese Lewis Rats. Experimental \& Clinical Endocrinology \& Diabetes 2009;117:473-9.

82. Oresic M, Simell S, Sysi-Aho M, Nanto-Salonen K, Seppanen-Laakso T, Parikka V, et al. Dysregulation of lipid and amino acid metabolism precedes islet autoimmunity in children who later progress to type 1 diabetes. Journal of Experimental Medicine 2008;205:2975-84.

83. Zhao X, Fritsche J, Wang J, Chen J, Rittig K, Schmitt-Kopplin P, et al. Metabonomic fingerprints of fasting plasma and spot urine reveal human pre-diabetic metabolic traits. Metabolomics 2010;6:36274.

84. Regulation of the activity of caspases by L-carnitine and palmitoylcarnitine. FEBS Letters 2000;478:19-25.

85. Palau-Rodriguez M, Tulipani S, Queipo-Ortuo MI, Urpi-Sarda M, Andres-Lacueva C. Metabolomic insights into the intricate gut microbial-host interaction in the development of obesity and type 2 diabetes. Frontiers in Microbiology 2015;6.

86. Shuo, Wang, Meiqin, Huang, Xue, You, et al. Gut microbiota mediates the anti-obesity effect of calorie restriction in mice. Scientific Reports 2018.

87. Hooper LV, Dan RL, Macpherson AJ. Interactions Between the Microbiota and the Immune System. Science 2012;336:1268-73.

88. Berg RD, Savage DC. Immune responses of specific pathogen-free and gnotobiotic mice to antigens of indigenous and nonindigenous microorganisms. Infection and Immunity 1975;11:320-9.

89. Wikoff WR, Kalisak E, Trauger S, Manchester M, Siuzdak G. Response and recovery in the plasma metabolome tracks the acute LCMV-induced immune response. Journal of proteome research 2009;8:3578-87.

90. Craig Stuart AS. Betaine in human nutrition. American Journal of Clinical Nutrition 2004:539-49.

91. Riley RT, An NH, Showker JL, Yoo HS, Norred WP, Chamberlain WJ, et al. Alteration of tissue and serum sphinganine to sphingosine ratio: an early biomarker of exposure to fumonisin-containing feeds in pigs. Toxicology \& Applied Pharmacology 1993;118:105-12. 
92. Luo ZF, Feng B, Mu J, Qi W, Wei Z, Guo YH, et al. Effects of 4-phenylbutyric acid on the process and development of diabetic nephropathy induced in rats by streptozotocin: Regulation of endoplasmic reticulum stress-oxidative activation. Toxicology \& Applied Pharmacology 2010;246:49-57.

93. Urquiza A, Liu S, Sjoberg M, Zetterstrom RH, Griffiths W, Sjovall J, et al. Docosahexaenoic Acid, a Ligand for the Retinoid X Receptor in Mouse Brain. Science 2000;290:2140-4.

94. Chen SJ, Hsu CP, Li CW, Lu JH, Chuang LT. Pinolenic acid inhibits human breast cancer MDA-MB-231 cell metastasis in vitro. Food Chemistry 2011;126:1708-15.

95. Gouesbet G, Jebbar M, Talibart R, Bernard T, Blanco C. Pipecolic acid is an osmoprotectant for Escherichia coli taken up by the general osmoporters ProU and ProP. Microbiology 1994;140 (Pt 9):2415.

96. Witt MR, Nielsen M. Differential modulation of brain benzodiazepine receptor subtypes by ricinelaidic acid in vitro. Biochemical Pharmacology 1994;47:742.

97. Pamela M, Jerusa B, Martín G. La microbiota intestinal: Un nuevo actor en el desarrollo de la obesidad. Revista Médica De Chile 2010;138:1020-7.

98. M., Fleming, T., Robinson, Thomson, B., et al. Global, regional, and national prevalence of overweight and obesity in children and adults during 1980-2013: a systematic analysis for the Global Burden of Disease Study 2013. LANCET -LONDON- 2014.

99. Dibner, J., J., Richards, J., D. Antibiotic Growth Promoters in Agriculture: History and Mode of Action. Poultry Science 2005;84:634-43.

100. Kabploy K, Bunyapraphatsara N, Morales NP, Paraksa N. Effect of Antibiotic Growth Promoters on Anti-oxidative and Anti-inflammatory Activities in Broiler Chickens. The Thai veterinary medicine 2016;46:89-95.

101. Genovese K. COMPETITIVE EXCLUSION AND COLONIZATION RESISTANCE AS ALTERNATIVES TO ANTIBIOTIC USE IN FOOD ANIMALS. Fish Physiology \& Biochemistry 2010;36:637-45.

102. A RL, B TZ, B AK, B ZL, B MS, B PF, et al. Feed-additive of bioengineering strain with surfacedisplayed laccase degrades sulfadiazine in broiler manure and maintains intestinal flora structure ScienceDirect. Journal of Hazardous Materials 2020.

103. Matsuda K, Tsuji H, Asahara T, Matsumoto K, Takada T, Nomoto K. Establishment of an analytical system for the human fecal microbiota, based on reverse transcription-quantitative PCR targeting of multicopy rRNA molecules. Appl Environ Microbiol 2009;75:1961-9.

104. Ornellas RMS, Santos TT, Arcucio LB, Sandes SHC, Oliveira MM, Dias CV, et al. Selection of lactic acid bacteria with probiotic potential isolated from the fermentation process of "Cupuaçu”(Theobroma grandiflorum). Advances in Microbiology, Infectious Diseases and Public Health: Springer, 2017:1-16.

105. A MC, a SMM-O, A EGM, B IB, a MM, A JG. Quantification of total bacteria, enterobacteria and lactobacilli populations in pig digesta by real-time PCR - ScienceDirect. Veterinary Microbiology 2006;114:165-70. 
106. Anthony, Bolger, Marc, Lohse, Bjoern, Usadel. Trimmomatic: a flexible trimmer for Illumina sequence data. Bioinformatics 2014.

107. Mago T, Salzberg SL. FLASH: Fast Length Adjustment of Short Reads to Improve Genome Assemblies. Bioinformatics 2011;27:2957-63.

108. QIIME allows analysis of high-throughput community sequencing data. Nature Methods 2010.

109. Benjamin J, Callahan, Paul J, McMurdie, Michael J, Rosen, et al. DADA2: High-resolution sample inference from Illumina amplicon data. Nature methods 2016.

110. Kindt R. BiodiversityR: Graphical User Interface for biodiversity and community ecology analysis. R package version 2.3 (2013-01-31). 2014.

111. Langille MGI, Zaneveld J, Caporaso JG, Mcdonald D, Knights D, Reyes JA, et al. Predictive functional profiling of microbial communities using 16S rRNA marker gene sequences. Nature Biotechnology 2013;31:814-21.

112. Evans A, Bridgewater B, Liu Q, Mitchell MW, Miller L. High Resolution Mass Spectrometry Improves Data Quantity and Quality as Compared to Unit Mass Resolution Mass Spectrometry in HighThroughput Profiling Metabolomics. Metabolomics 2014;4.

113. Jonas Z, Jackson MA, Gabi K, Massimo M, Tao L, Amalio T, et al. The fecal metabolome as a functional readout of the gut microbiome. Nature Genetics 2018;50:790-5.

114. Cui L, Lee YH, Kumar Y, Xu F, Lu K, Ooi EE, et al. Serum metabolome and lipidome changes in adult patients with primary dengue infection. PLoS neglected tropical diseases 2013;7:e2373.

115. Bar N, Korem T, Weissbrod O, Zeevi D, Segal E. A reference map of potential determinants for the human serum metabolome. Nature 2020;588:135-40.

116. Wold V, Antti H, Lindgren F, Öhman J. Orthogonal signal correction of near-infrared spectra. Chemometrics and Intelligent Laboratory Systems 1998.

117. Chen J, Zhao X, Fritsche J, Yin P, Schmitt-Kopplin P, Wang W, et al. Practical approach for the identification and isomer elucidation of biomarkers detected in a metabonomic study for the discovery of individuals at risk for diabetes by integrating the chromatographic and mass spectrometric information. Analytical Chemistry 2008;80:1280-9.

118. Lazar AG, Romanciuc F, Socaciu MA, Socaciu C. Bioinformatics Tools for Metabolomic Data Processing and Analysis Using Untargeted Liquid Chromatography Coupled With Mass Spectrometry. Bulletin of University of Agricultural Sciences \& Veterinary Medicine Cluj Napoca Animal Science \& Biotechnologies 2015;72.

\section{Figures}


A

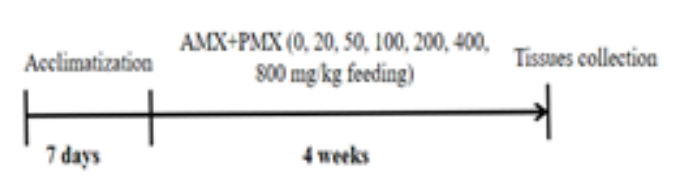

B

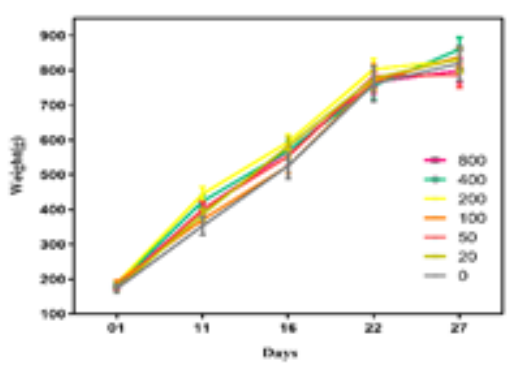

Figure 1

Growth performance of broiler chicken under the feed-additive regimens of AMX+PMX antibiotics. (A) Experimental design. (B) The body weight curve of chicken during feeding experiment. Data show the mean $\pm S D(n=10$ chicken per group $)) . * *=P \otimes 0.01 ; * \star \star \star ~=P \otimes 0.0001$
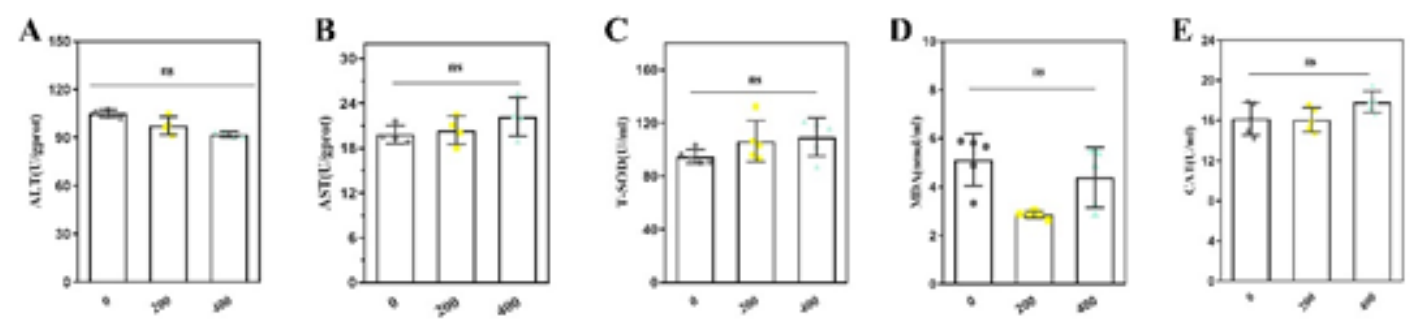

$\mathbf{F}$
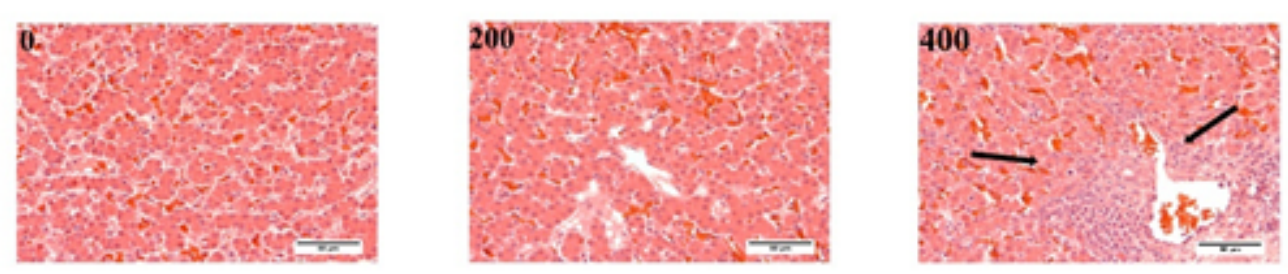

G
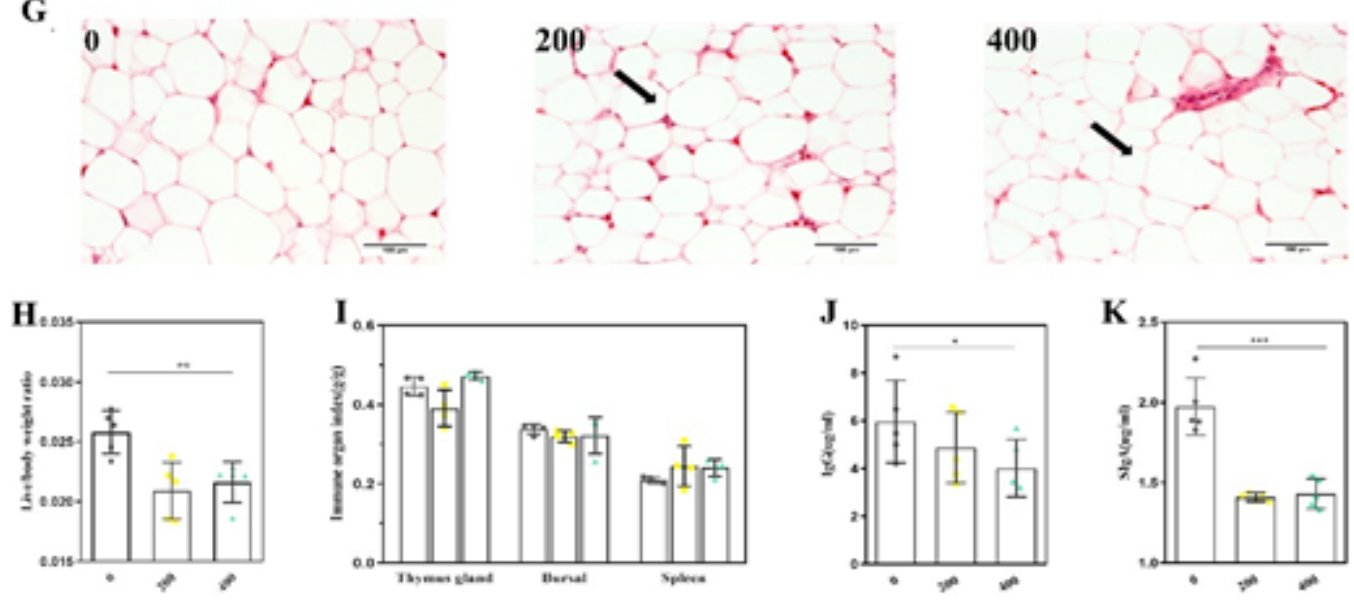

Figure 2

Effect of antibiotics on chicken immune organ, live and adipose tissue. ALT level (A), AST (B), T-SOD activities (C), MDA (D), and CAT (E) were detected in 0, 200 and 400 groups. Histological analysis of liver 
$(F)$ and adipocyte $(\mathrm{G})$ under a microscope has been shown. $(\mathrm{H})$ Comparison of three immune organ indexes from the thymus gland, bursa, and spleen. $(\mathrm{I}, \mathrm{J})$ Comparison of serum IgG and intestinal SIgA levels among the three groups. (K) Liver/body weight mass ratio in different groups. For A-E and $\mathrm{H}-\mathrm{K}$ values are expressed as the mean $\pm S D(n=5)$. For $F-G, n=3$. Statistical analyses were conducted using the one-way ANOVA. ${ }^{*} p<0.05,{ }^{*} p<0.01,{ }^{* *} p<0.001,{ }^{* \star * *} p<0.0001$; ns: not significant.
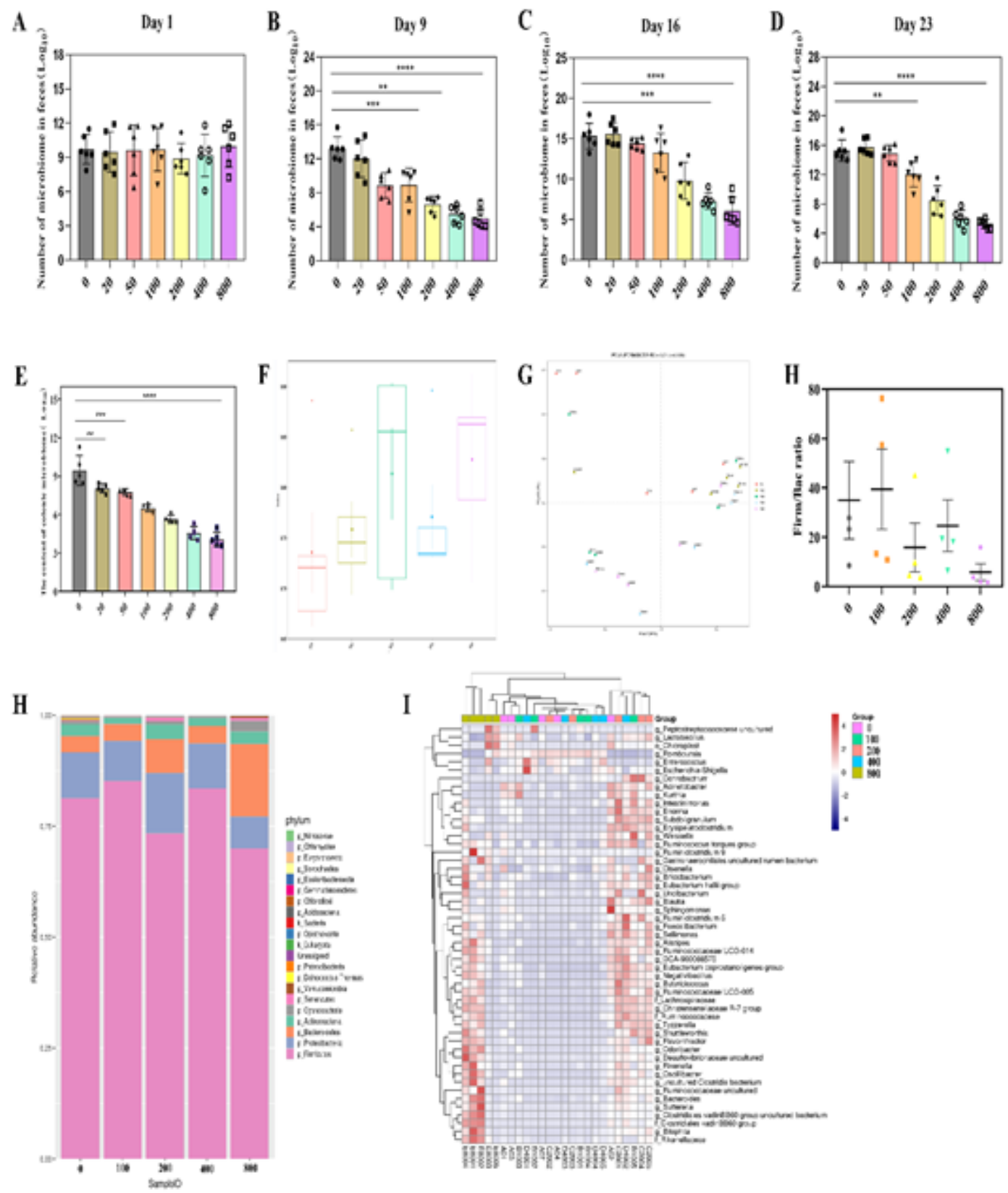

Figure 3

Effects of $A M X+P M X$ antibiotics treatment on gut microbiota of chicken. (A-D) The number of gut microbes in feces determined by QRT-PCR on day 1,9,16 and 23. (E) The proportion of gut microbes in the colon determined by RT-PCR on day 35th. (F) Bacterial diversity as assessed by the Shannon index. (G) Scatter plot of the principal coordinate analysis (PCoA) scores depicting variance in broiler intestinal 
microflora. $(\mathrm{H})$ The Firmicutes/Bacteroidetes ratio in the different groups. (I) Comparison of phylum relative abundance in different groups. $(\mathrm{J})$ The relative abundance of the most abundant bacteria genera in the four groups. Different colors indicate the biomarkers identified in different groups. Bars show the mean \pm SD ( $n=5$ chicken per group). ${ }^{* *}=P \otimes 0.01 ; * \star \star \star *=P \otimes 0.0001$.

A
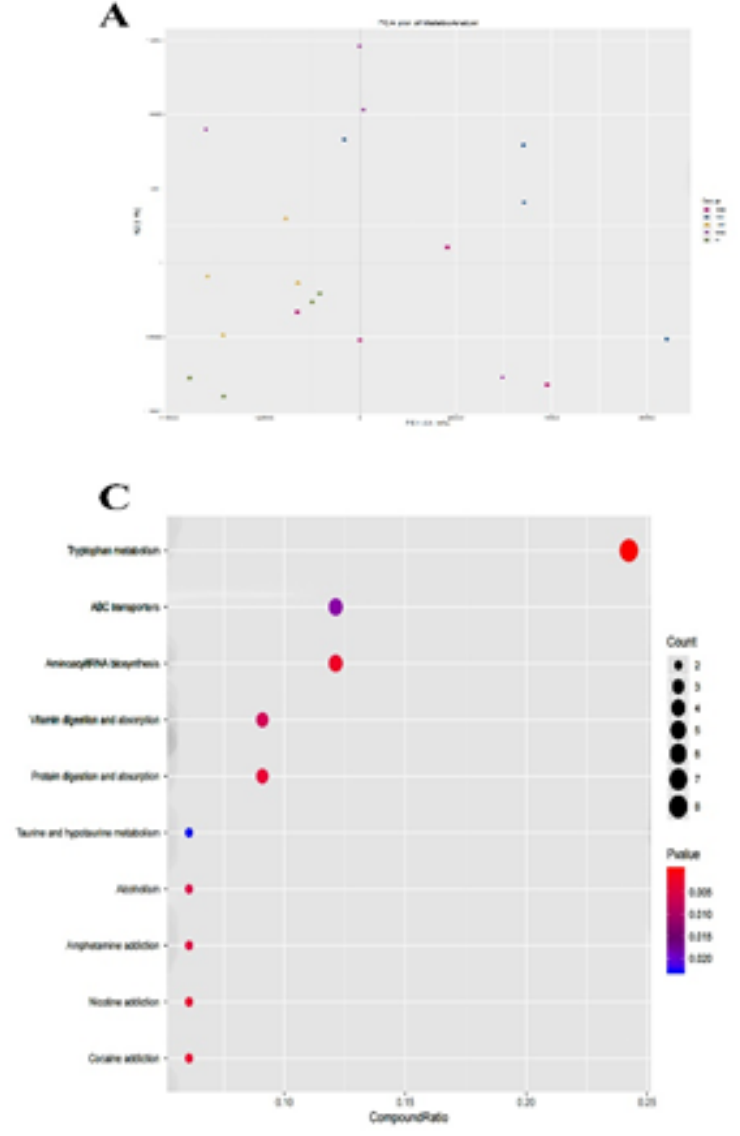

B

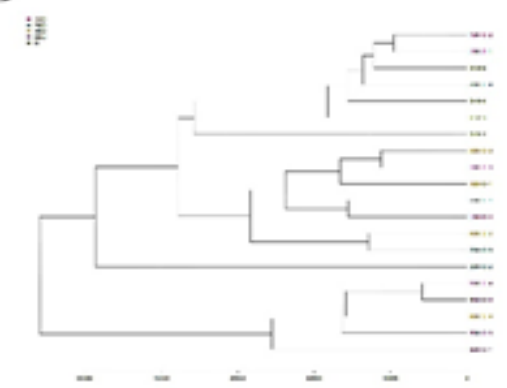

D

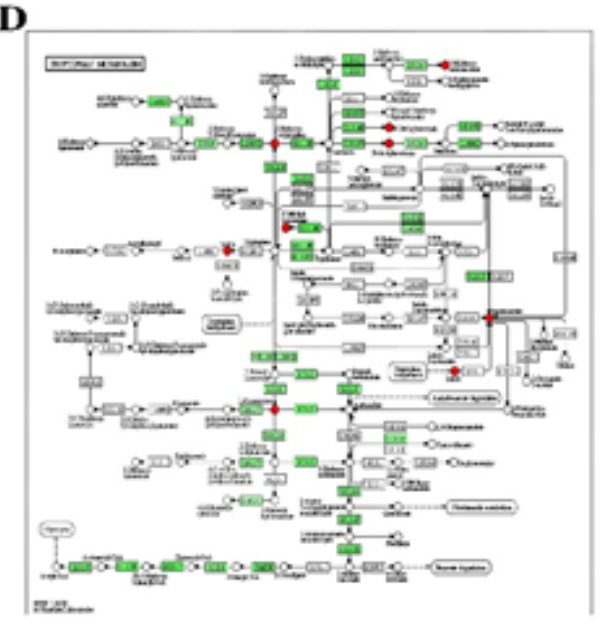

Figure 4

Microbiome metabolome of chicken feces. (A) PCA of all groups based on metabolomics data. (B) Drawing of evolutionary tree based on sample normalization abundance. (C) KEGG analysis of metabolites according to the selected differential metabolites and KEGG annotation ID. The abscissa is the proportion of metabolites and the ordinate is the pathway. The red the dot is the $\mathrm{P}$ value, and the size of the dot is the mount of metabolites. (D) Tryptophan metabolism pathway illustration using KEGG. The red dot is showed the site of different substances. 


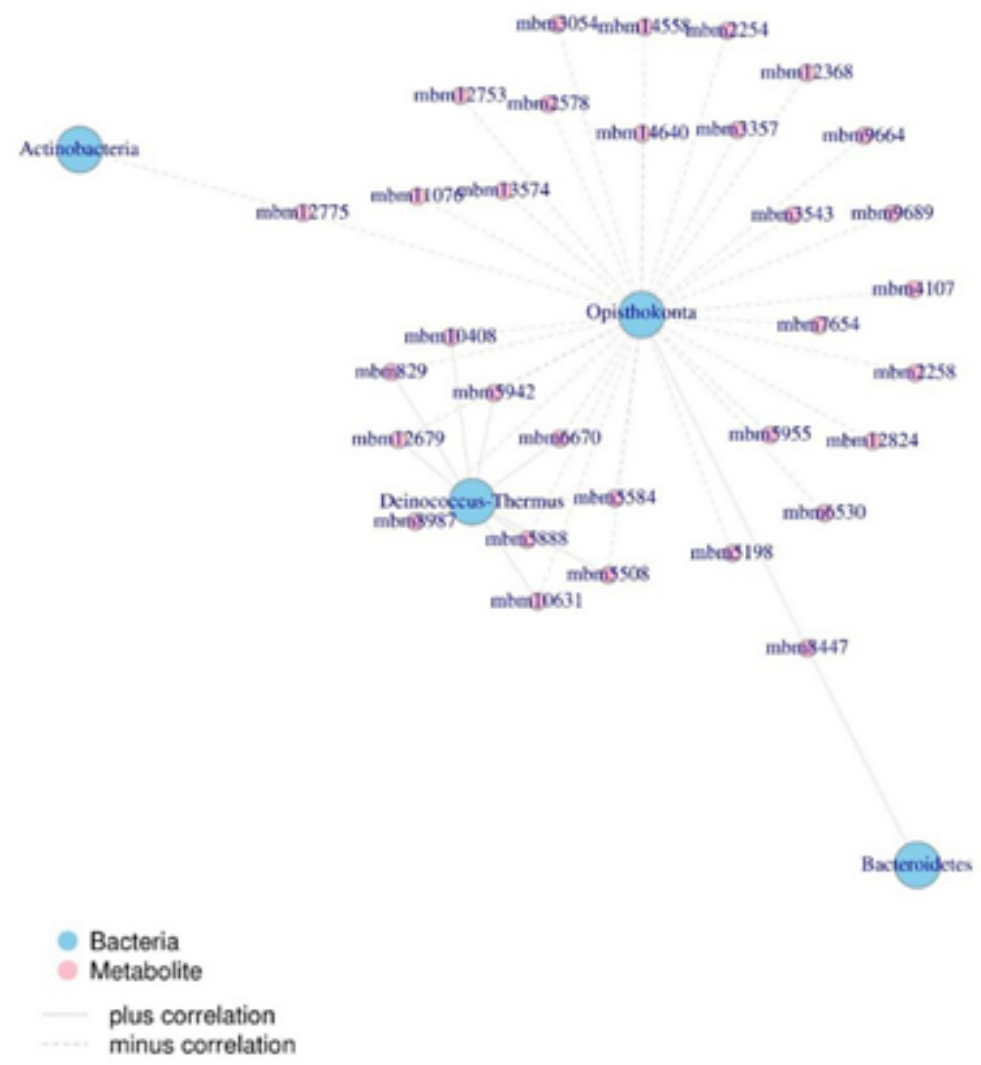

Figure 5

Network revealed the role of antibiotic and bacterial metabolomic correlation in intestinal microbiota. 

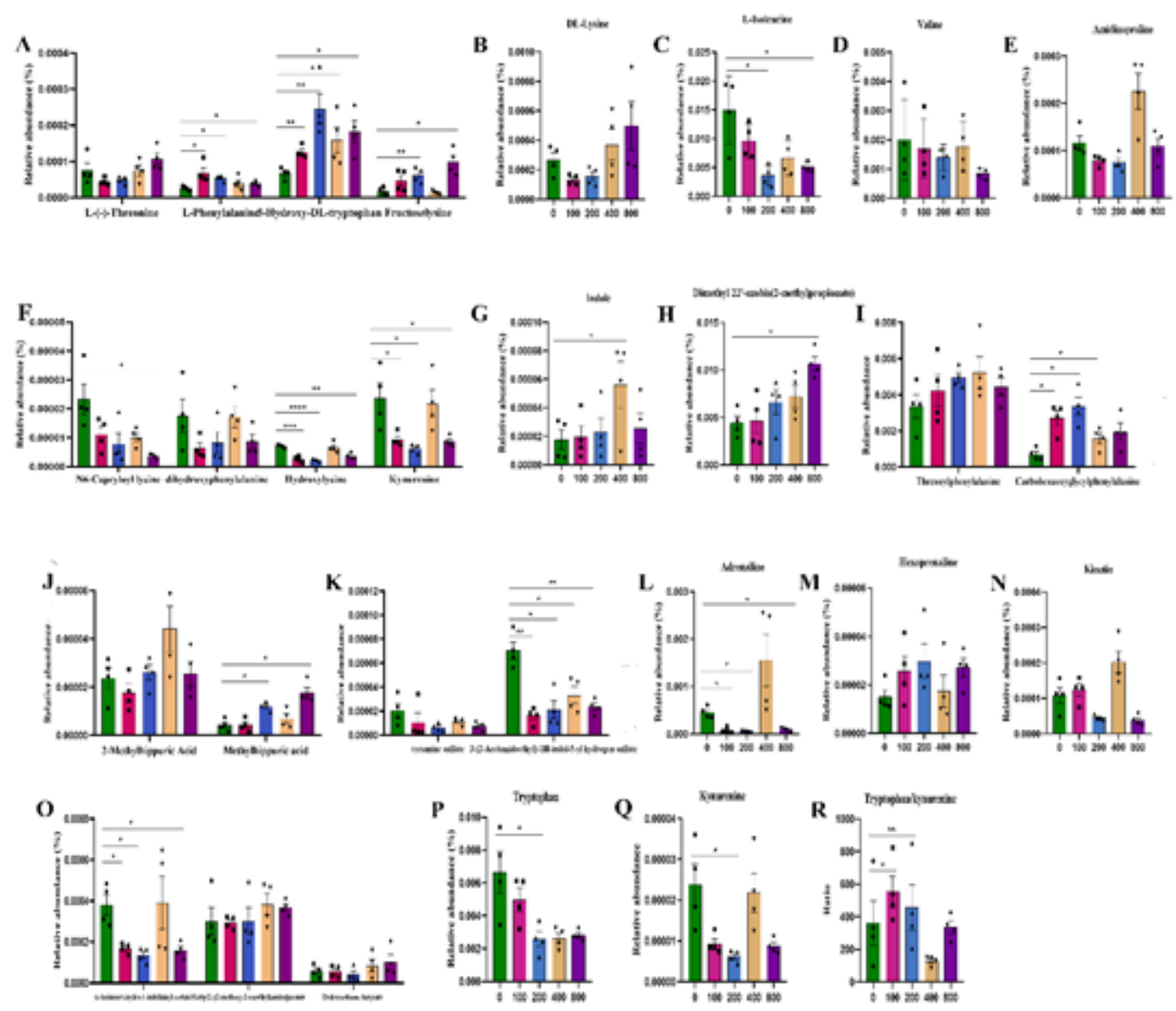

Figure 6

Metabolic alterations observed in fecal samples. (A) D L-(-)-Threonine, L-Phenylalanine, 5-Hydroxy-DLtryptophan, Fructoselysine. (B)DL-Lysine. (C) L-Isoleucine. (D) Valine. (E) Amidinoproline. (F) N6-Capryloyl lysine, dihydroxyphenylalanine, hydroxylysine, kynurenine. (G) Indole. (H) Dimethyl 22'-azobis(2methylpropionate). (I) Threonylphenylalanine, Carbobenzoxyglycylphenylalanine. (J) 2-methylhippuric acid, methylhippuric acid. (K) tyramine sulfate, 3-(2-Acetamidoethyl)-1H-indol-5-yl hydrogen sulfate. (L) Adrenaline. (M) Hexoprenaline. (N) Kinetin. (O) 6-Aminooctahydro-1-indolizinyl acetate, methyl 2-[(2methoxy-2-oxoethyl) amino] acetate, hydrocortisone butyrate (JP15/USP). (P) Tryptophan. (Q)

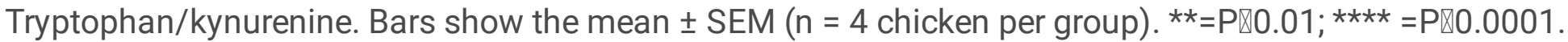



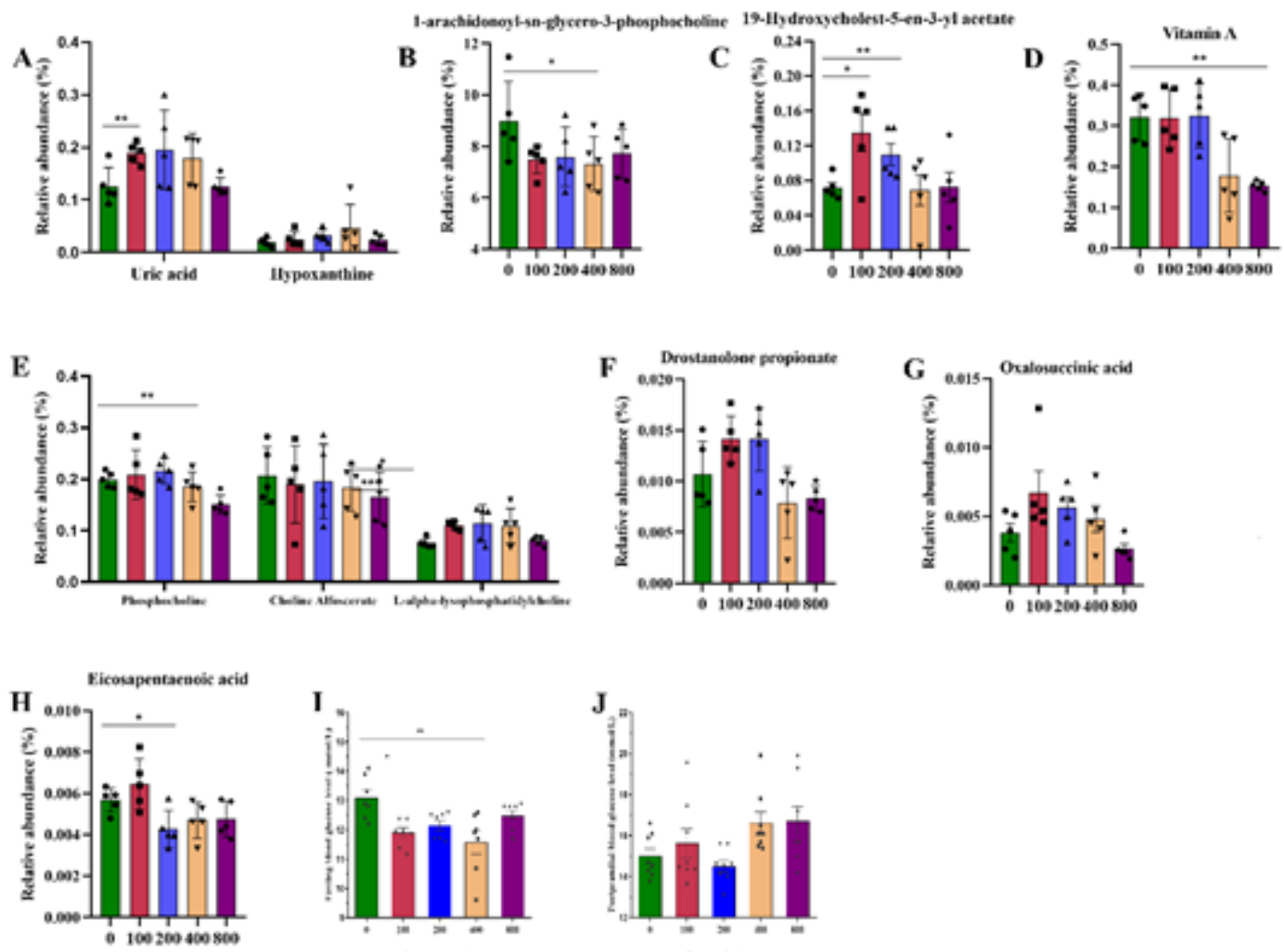

Figure 7

Serum metabolites altered in feed antibiotics as compared with controls. Different colors indicate the biomarkers identified in different groups. (A) Uric acid and hypoxanthine. (B) 1-arachidonoyl-sn-glycero-3phosphocholine. (C) 19-Hydroxycholest-5-en-3-yl acetate. (D) Vitamin A. (E) Phosphocholine, L-alphalysophosphatidylcholine and choline alfoscerate. (F) Drostanolone propionate. (G) Eicosapentaenoic acid. $(\mathrm{H})$ Oxalosuccinic acid. Blood glucose levels an hour before (I)or after $(\mathrm{J})$ dinner. Bars show the mean \pm SEM ( $n=5$ chicken per group). ** $=P \llbracket 0.01 ; * \star \star \star ~=P \unrhd 0.0001$. 

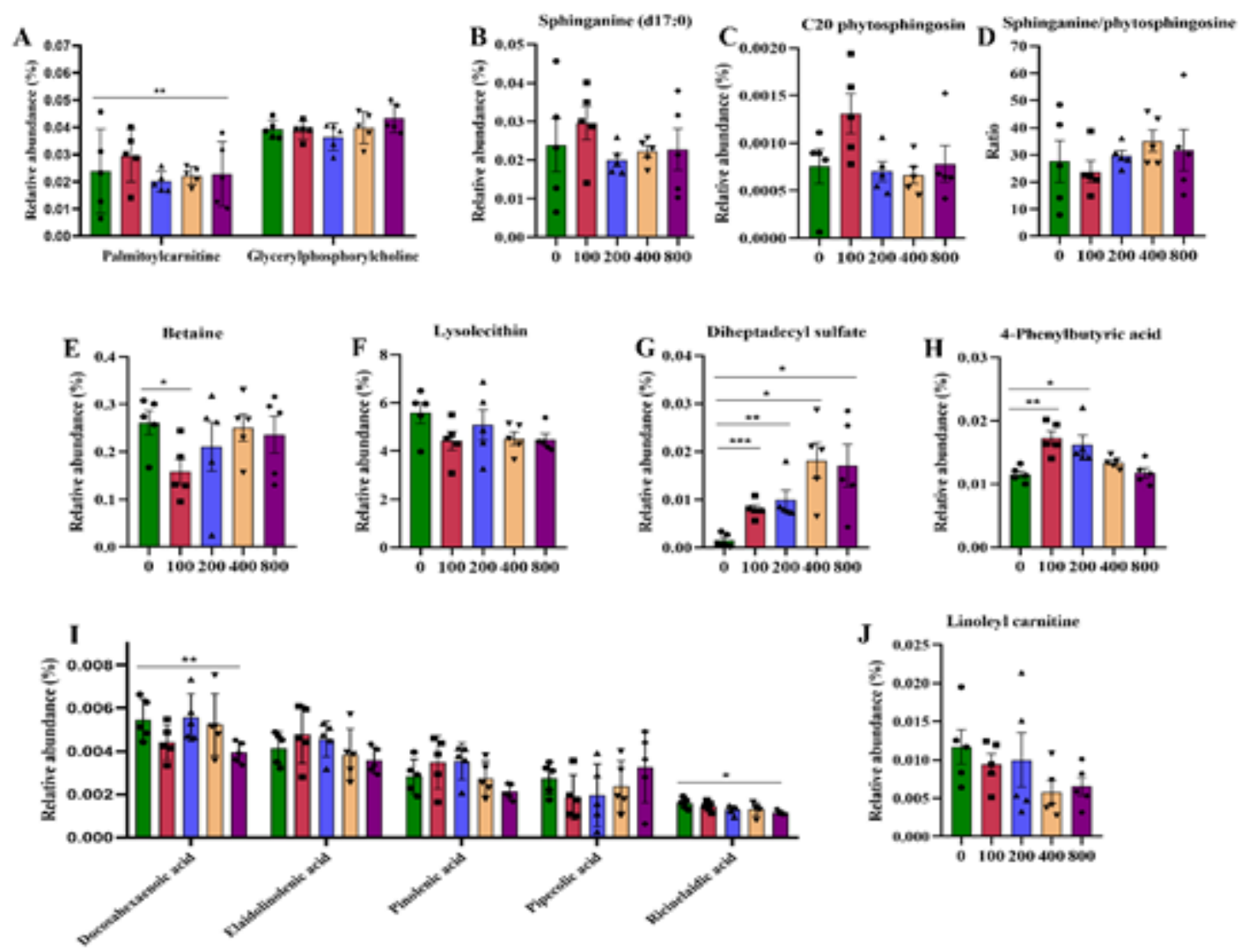

Figure 8

Metabolism production of the antibiotic-induced immune response in serum lipidomic. Typical change trends based on differential metabolite classes in serum. Different colors indicate the biomarkers identified in different groups. (A) Palmitoylcarnitine and glycerylphosphorylcholine. (B) Sphinganine. (C) C20 phytosphingosine. (D) Sphinganine / C20 phytosphingosine. (E) Betaine. (F) Lysolecithin. (G) Diheptadecyl sulfate. (H) 4-Phenylbutyruc acid. (I) Docosahexaenoic acid, elaidolinolenic acid, pinolenic acid, pipecolic acid and ricinelaidic acid. (J) Linoleyl carnitine. Bars show the mean \pm SEM $(n=5$ chicken per group) $\star \star=P \otimes 0.01 ; \star \star \star \star ~=P \otimes 0.0001$.

\section{Supplementary Files}

This is a list of supplementary files associated with this preprint. Click to download.

- Supplementalfile1.docx

- Supplementalfile2.xlsx

- Supplementalfile3.docx 